\title{
Insulin-taking behavior and memory problems among people with diabetes in five countries: findings from a web survey
}

This article was published in the following Dove Press journal:

Patient Intelligence

10 November 2014

Number of times this article has been viewed

\author{
Meryl Brod' \\ Kathryn M Pfeiffer ${ }^{\prime}$ \\ Kasper Mandel Clausen ${ }^{2}$ \\ Rasmus Skovgaard ${ }^{3}$ \\ Jens Harald Kongs $\varnothing^{2}$ \\ 'The Brod Group, Mill Valley, CA, \\ USA; ${ }^{2}$ Novo Nordisk A/S, Global \\ Development, Søborg, Denmark; \\ ${ }^{3}$ Novo Nordisk Canada Inc., \\ Market Access and External Affairs, \\ Mississauga, ON, Canada
}

Correspondence: Meryl Brod

The Brod Group, 219 Julia Ave, Mill Valley, CA 9494I, USA

$\mathrm{Tel}+|4| 538 \mid 5532$

Fax + I 4I5 38I 0653

Email mbrod@thebrodgroup.net
Background: The purpose of this study was to explore memory problems that result in nonadherence with insulin treatment in people with diabetes from five countries (USA, UK, Canada, Germany, People's Republic of China), as well as the impact of memory problems on physical and emotional functioning, work, and diabetes management.

Methods: A web survey was conducted in five countries with diabetic patients taking insulin. In three countries, focus groups and interviews with 64 patients treated with insulin were used to develop survey items. Three types of memory problems associated with insulin-taking were examined: unintentionally forgetting (UF), questioning whether or not one took a dose (QT), and questioning how much was taken (QD) over the past month. The survey focused on respondents who had experienced at least one of these memory problems.

Results: The incidence of memory problems was high; $93.3 \%$ of those eligible for the survey reported having had a memory issue in the previous month. A total of 1,754 patients with type 1 or type 2 diabetes completed the web survey. Sixty-eight percent of respondents reported a UF, 79.6\% a QT, and 54.7\% a QD. A total of 35.9\% of respondents reported experiencing all three memory problems. Respondents reported that memory problems had negative impacts on physical and emotional functioning, as well as time and functioning at work. Many respondents indicated that memory problems led them to taking additional blood glucose measurements and contacting physicians/health care professionals. The results demonstrated some significant differences across countries, some significant variation across patient demographics and disease characteristics, and few significant differences across types of insulin regime.

Conclusion: Memory problems related to insulin-taking among people with diabetes were experienced by almost all patients. Memory problems are associated with poorer patient wellbeing and functioning, increased use of health care resources, lost time, and reduced functioning at work. Memory problems should be considered in diabetes treatment in order to improve insulin adherence, diabetes management, and patient outcomes.

Keywords: insulin, memory problems, diabetes, web survey

\section{Introduction}

Medication nonadherence among diabetes patients is a well-known clinical challenge in diabetes management, especially for patients on insulin. ${ }^{1,2}$ Although measures and estimates of insulin nonadherence vary, studies suggest that insulin nonadherence is quite prevalent, with estimates ranging from approximately one third to two thirds of patients. ${ }^{1-7}$ Among patients with type 1 (T1D) or type 2 (T2D) diabetes treated with insulin in eight countries, research indicates that $35 \%$ reported insulin nonadherence one or more times in the previous month, with an average of 3.4 days of nonadherence reported over the previous month among those nonadherers. ${ }^{6}$ 
Insulin nonadherence is clinically relevant because research has consistently shown that lack of adherence to prescribed insulin regimens is related to poorer health outcomes, and better adherence is related to better levels of glycemic control. ${ }^{4,8,9}$ Additionally, research shows that insulin nonadherence is associated with increased risk of mortality, even after controlling for factors known to increase mortality rates, such as age, body mass index, cholesterol, blood pressure, and smoking status. ${ }^{10}$ Insulin nonadherence is also associated with increased hospital admissions due to diabetic ketoacidosis and other acute complications of diabetes. ${ }^{11}$

While nonadherence due to intentional missing of insulin and intentional dose adjustments is well documented, ${ }^{3,7}$ less is understood about the prevalence or consequences of memory problems (MPs) related to unintentional nonadherence. Unintentional nonadherence may be due to several factors or MPs, including forgetting to have insulin available when needed as well as simply forgetting to take the insulin at the correct time or at the correct dose. A greater understanding of these unintentional MPs may help facilitate improved diabetes treatment management strategies and reduce the negative clinical consequences of unintentional nonadherence.

The purpose of this web-based survey was to examine the frequency and characteristics of unintentional MPs among diabetes patients taking insulin in order to better understand reasons for MPs, patient concerns, and responses to MPs. Further, the web survey aimed to investigate the consequences of unintentional nonadherence due to MPs on diabetes management, patient functioning and well-being, and health care contacts.

\section{Materials and methods}

The web-based survey was conducted in five countries (USA, UK, Canada, Germany, People's Republic of China). The analyses present results for the overall sample and by country.

\section{Survey development}

To generate items for the survey, focus groups and interviews were conducted in the People's Republic of China, Canada, and Germany with diabetes patients taking insulin in order to understand MPs from a patient perspective. Transcripts from the focus groups were qualitatively analyzed, based on grounded theory principles, to derive themes and major areas of importance regarding MPs. Survey items were then generated based on the qualitative analysis, and items were cognitively debriefed for comprehension and usability to ensure flow and logic of the web survey.

\section{Participants}

The survey was conducted online with respondent recruitment through patient panels and invites via email. Initial invites were sent to those preidentified as having diabetes (and confirmed via screening). As fielding of the survey progressed, invites were sent to the general population. Respondents received a minimal honorarium for their participation in the survey. To be eligible for the survey, respondents had to be at least 18 years of age, have previously been diagnosed with diabetes by a doctor or health care professional, know whether they are diagnosed with T1D or $\mathrm{T} 2 \mathrm{D}$, and take insulin using vial and needle or syringe or by an insulin pen-injector, either prefilled or durable. Patients using insulin pumps were excluded as it was felt that the pump may introduce a delivery system bias. Respondents who only took fast-acting insulin or had medical conditions known to affect memory such as dementia were excluded. Recruitment quotas were established for type of insulin regime (basal only, basal bolus, and premix), age, type of diabetes, and country to ensure adequate sampling across groups. Each respondent in the survey received a unique identification number. The study was approved by the Copernicus Group institutional review board (TBG1-12082) in March 2012.

\section{Survey variables}

All responses to the survey were self-reported, and assessed the items described in the following sections. Demographic variables included country, age, sex, marital status, educational level, whether working for pay, number of work hours per week, and an ordinal measure of the degree to which the respondent has trouble remembering in general. Additionally, characteristics related to diabetes including age of diagnosis, type of diabetes, age at initiation on an insulin regimen, insulin injection method, and whether or not the subject was taking oral antidiabetic drugs were collected.

Variables concerning the incidence and prevalence of MPs included whether or not each type of MP occurred in the past month and ordinal measures of the frequency of MPs (never, very rarely, rarely, sometimes, often, very often).

Variables concerning the patient experience of MPs included when and where MPs occurred, concerns patients had regarding MPs, reasons given for MPs, and how patients realized MPs had occurred. Patient confidence in dealing with MPs, measured using an ordinal scale, was also collected. Additionally, the survey measured respondent use of strategies or visual cues to help them know if the appropriate dose was taken when questioning whether 
or not one took a dose (QT), and questioning how much was taken (QD).

Concerns about MP variables included the patient's degree of worry (including double dosing, hyperglycemia, and hypoglycemia), which was assessed on a 10-point scale. Respondents were asked to indicate their degree of worry regarding such factors using numbers ranging from 0 to 10 , where 0 indicated "not at all worried" and 10 indicated "extremely worried". Such a scale allowed for a more sensitive assessment of degree of worry compared with dichotomous (eg, yes/no) or categorical responses.

Variables concerning the corrective action taken following MPs included the patient's response to or corrective action taken following the MP, which included either measuring blood glucose then taking insulin based on the reading, taking an insulin dose without measuring blood glucose, or skipping the insulin dose (doing nothing).

Variables concerning the consequences of MPs included recovery time following MPs, which was measured by the time taken to return to normal physical and emotional functioning, as well as time taken to return to normal blood glucose levels and time to resolve symptoms of hyperglycemia. Also assessed was the impact of MPs on physical and emotional functioning at work. These items were also scored on a 10-point scale, where 0 indicated "no impact" and 10 indicated "an extremely negative impact". Respondents were also asked about the number of additional blood glucose measurements taken as a result of the MP in both the 24-hour and 7-day periods following the MP. Additionally, patientreported measures of actual missed work time were captured, including being late for work, leaving work early, missing meetings/appointments, and missing a full day of work following an MP. Whether or not respondents experienced hyperglycemia following an MP was also assessed.

Variables concerning the experience with health care professionals included whether or not the respondent visited a physician or other health care professional due to MP and whether or not the respondent contacted (via telephone or email) a physician or other health care professionals as a result of the MP.

\section{Data analysis}

Analyses of the survey data included descriptive statistics (means, standard deviations, percentages, and ranges) and measures of association, including comparison of means and cross tabulations. Statistical significance tests were conducted as appropriate. One-way analysis of variance was used for comparing means, and Pearson's chi-square was used for analyzing associations between categorical variables. Due to a small number of outlier values for questions regarding recovery time (time taken to return to normal blood glucose level, time for high blood glucose symptoms to resolve, and time to return to usual physical and emotional functioning following MP), data used in the analyses for these questions were trimmed by $5 \%$ at the upper level to exclude probable reporting errors.

All analyses were conducted on the sample as a whole, as well as for each individual country, to explore possible cross-country differences. For each MP, the study investigated whether or not key findings varied by age, type of diabetes, and duration of diabetes because these are important demographic and disease factors that may influence findings. Specifically, analyses were conducted to examine whether or not any of these factors were associated with variation in frequency of MPs, corrective actions taken following MPs (measuring blood glucose and taking dose based on reading, taking insulin without measuring blood glucose, or skipping insulin dose), any missed work time resulting from MPs, and health care contact related to MPs. Differences in results by type of insulin regime were also examined.

\section{Results}

\section{Survey development}

A total of 64 persons with diabetes participated in seven semistructured focus groups and eight telephone interviews in three countries, ie, Canada $(n=24)$, the People's Republic of China $(n=24)$, and Germany $(n=16)$. Focus groups and interviews were conducted in each respondent's native language and then translated into English as necessary.

Fifty percent of the focus group respondents were male and $67.2 \%$ were married. The mean age of the sample was 50.1 (range 18-72) years. Fifty percent of the sample worked full-time for pay, $12.5 \%$ worked part-time for pay, and $28.1 \%$ were retired or not working. A total of $4.7 \%$ were disabled and unable to work and $4.7 \%$ were students. A total of $60.9 \%$ of participants had post secondary or undergraduate education, or beyond. In terms of diabetes type, $34.4 \%$ had T1D and a $65.6 \%$ had T2D. The mean age at initiation on an insulin regimen was 41.2 (range 6-64) years. On average, patients took 2.6 (range 1-8) injections of insulin per day.

Based on qualitative analysis of the focus group and interview transcripts, MPs were classified as: unintentionally forgetting (UF), eg, due to not having insulin available when forgetting to bring it to a restaurant, QT or QD. These were defined in the survey as follows: 
- UF - "you were sure you missed (did not take) an insulin dose; you did not question whether or not you missed your dose. This was not deliberate or intentional and may be because you didn't have your insulin or pen/needles/vial with you or they were not available."

- QT - "you questioned yourself, or were unsure, about whether or not you took an insulin dose."

- QD - "you questioned yourself, or were unsure, about how much insulin you took" (the dose amount).

The major themes identified from the qualitative analysis were:

- incidence and prevalence of MPs

- the patient's experience of MPs

- concerns about MPs

- corrective action taken following MPs

- consequences of MPs

- experiences with health care professionals.

Item generation for the survey was based on capturing the details of each of these themes. The survey was debriefed in seven patients to ensure that respondents understood the questions as intended and to ensure that the electronic version of the survey worked properly. Surveys were administered in the native language of each country.

\section{Survey findings}

A total of 11,711 potential eligible respondents with diabetes were approached for the web survey (Table 1). Of these, 10,924 respondents had reported some problems with missing insulin doses in the previous month due to MPs and were eligible to continue with the survey. Due to country recruitment quotas for demographic/diabetes characteristics, 9,170 eligible patients were removed from the sample before completing the survey, resulting in an analytic sample of 1,754 patients with diabetes who had experienced at least one MP in the previous month (People's Republic of China, $\mathrm{n}=354$; Germany, $\mathrm{n}=350$; USA, $\mathrm{n}=350$; UK, $\mathrm{n}=350$; and Canada, $\mathrm{n}=350$ ).

The survey sample statistics, including demographics, diabetes characteristics, and MPs, are shown in Table 2. Briefly, the average age of the respondents was 40.0 (range 18-99) years, $54.5 \%$ were male, and $73.4 \%$ worked for pay. Among all respondents, $15.2 \%$ indicated that they experienced moderate or substantial forgetfulness in general.

A total of $59.7 \%$ of respondents had T1D and the mean age of first taking insulin was 31.6 years. A total of $30.7 \%$ of respondents used a vial/syringe for insulin injections, while $69.3 \%$ used a prefilled pen-injector. A total of $56.2 \%$ of respondents reported that their diabetes was very well or well controlled (compared with moderately controlled, poorly controlled, or very poorly controlled), and $67.7 \%$ indicated that their health was good, very good, or excellent (compared with fair or poor).

\section{Prevalence of memory problems}

The prevalence of any type of MP in the past month among those approached and eligible for the survey (eligible, $\mathrm{n}=11,711$; MP, $\mathrm{n}=10,924$ ) was $93.3 \%$. In the analytic sample $(n=1,754), 68.0 \%$ of respondents reported that, in the past month, they had a UF, 79.6\% had a QT, and 54.7\% had a QD. A total of $66.5 \%$ reported having experienced at least two of the MPs, while $35.9 \%$ had experienced all three types of MP in the past month. Analyses revealed significant cross-country differences in the incidence of MPs (Figure 1).

Respondents also reported on the frequency with which they experienced MPs. Slight variation in the frequency of MPs was evident across countries. Overall, the pattern of country variation was similar across MPs; however, this variation was statistically significant for UF $(P<0.001)$ and QT $(P<0.01)$ but not for QD. Table 3 shows the frequency of MPs for the full sample and by country.

Table I Survey flowchart

\begin{tabular}{|c|c|c|c|c|c|c|c|}
\hline & $\begin{array}{l}\text { People's } \\
\text { Republic of } \\
\text { China }\end{array}$ & Germany & USA & UK & Canada & Total & $\begin{array}{l}\text { Percentage } \\
\text { of total } \\
\text { approached }^{\mathrm{a}}\end{array}$ \\
\hline All eligible respondents with diabetes ${ }^{a}$ & 3,083 & 3,377 & $\mathrm{I}, \mathrm{I} 46$ & $\mathrm{I}, 844$ & 2,261 & $|I, 7| \mid$ & $34.1 \%$ \\
\hline $\begin{array}{l}\text { Respondents with diabetes who have no } \\
\text { memory problems }\end{array}$ & 290 & 178 & 118 & 143 & 58 & 787 & $2.3 \%$ \\
\hline $\begin{array}{l}\text { Remaining eligible respondents with diabetes and at } \\
\text { least one memory problem (either UF, QT, or QD) }\end{array}$ & 2,793 & 3,199 & 1,028 & $\mathrm{I}, 70 \mathrm{I}$ & 2,203 & 10,924 & $31.8 \%$ \\
\hline Prevalence of respondents with memory problems & $90.6 \%$ & $94.7 \%$ & $89.7 \%$ & $92.2 \%$ & $97.4 \%$ & $93.3 \%$ & \\
\hline Quota full (removed) & 2,443 & 2,849 & 678 & $\mathrm{I}, 35 \mathrm{I}$ & 1,849 & 9,170 & $26.7 \%$ \\
\hline Total in analysis & 350 & 350 & 350 & 350 & 354 & $\mathrm{I}, 754$ & $5.1 \%$ \\
\hline
\end{tabular}

Note: ${ }^{a}$ Respondents approached to complete survey, total 34,389; respondents removed because had no diagnosis of diabetes, total 12,929 ; remaining respondents with diabetes, total 21,460; noneligible respondents with diabetes removed for reasons other than memory problems, total 9,749.

Abbreviations: UF, unintentionally forgetting; QT, questioning whether or not one took dose; QD, questioning how much was taken. 
Table 2 Demographics, diabetes, and memory statistics $(n=I, 754)$

\begin{tabular}{|c|c|c|c|c|c|c|}
\hline & $\begin{array}{l}\text { People's } \\
\text { Republic of China } \\
(n=354)\end{array}$ & $\begin{array}{l}\text { Germany } \\
(n=350)\end{array}$ & $\begin{array}{l}\text { USA } \\
(n=350)\end{array}$ & $\begin{array}{l}\text { UK } \\
(n=350)\end{array}$ & $\begin{array}{l}\text { Canada } \\
(n=350)\end{array}$ & $\begin{array}{l}\text { Total } \\
n=I, 754\end{array}$ \\
\hline \multicolumn{7}{|l|}{ Demographic characteristics } \\
\hline \multicolumn{7}{|l|}{$\mathrm{Age}^{\mathrm{b}}$} \\
\hline Mean (SD) & $43.2(13.4)$ & $39.5(13.3)$ & $44.3(16.6)$ & $40.3(14.3)$ & $37.1(13.2)$ & $40.0(14.4)$ \\
\hline Range & $25-70$ & $18-99$ & $18-75$ & $18-79$ & $18-74$ & $18-99$ \\
\hline \multicolumn{7}{|l|}{ Sex ${ }^{a}$} \\
\hline n (\%) Male & $209(59.0)$ & $214(6 I . I)$ & $179(5 \mid .1)$ & $172(49.1)$ & $182(52.0)$ & $956(54.5)$ \\
\hline \multicolumn{7}{|l|}{ Marital status ${ }^{\mathrm{b}}$} \\
\hline n (\%) Married/partnered & $308(87.0)$ & $250(71.5)$ & $174(49.7)$ & $244(69.8)$ & $188(53.7)$ & $\mathrm{I}, \mathrm{I} 64(66.3)$ \\
\hline $\mathrm{n}(\%)$ Single & $35(9.9)$ & $77(22.0)$ & $107(30.6)$ & $86(24.6)$ & $138(39.4)$ & $443(25.3)$ \\
\hline \multicolumn{7}{|l|}{ Education ${ }^{\mathrm{b}}$} \\
\hline n (\%) High school & $35(9.9)$ & $156(44.6)$ & $130(37.1)$ & I0I (28.9) & $124(28.9)$ & $546(31.1)$ \\
\hline n (\%) College/grad school & $319(90.1)$ & $191(54.6)$ & $220(62.9)$ & $225(64.3)$ & $223(63.7)$ & $\mathrm{I}, \mathrm{I} 78(67.2)$ \\
\hline \multicolumn{7}{|l|}{ Work for pay ${ }^{\mathrm{b}}$} \\
\hline n (\%) Yes & $313(88.4)$ & $288(82.3)$ & $174(49.7)$ & $270(77.1)$ & $243(69.4)$ & I,288 (73.4) \\
\hline \multicolumn{7}{|l|}{ Hours worked ${ }^{b}$} \\
\hline Mean (SD) & $39.6(6.2)$ & $39.0(7.1)$ & $36.2(10.6)$ & $34.9(9.8)$ & $38.4(12.1)$ & $37.8(9.3)$ \\
\hline \multicolumn{7}{|l|}{ General trouble remembering } \\
\hline n (\%) Moderate/substantial & $44(12.4)$ & $20(5.7)$ & $74(21.1)$ & $54(15.4)$ & $74(21.1)$ & $266(15.2)$ \\
\hline \multicolumn{7}{|l|}{ Diabetes characteristics } \\
\hline \multicolumn{7}{|l|}{ Age diagnosed ${ }^{\mathrm{b}}$} \\
\hline Mean (SD) & $36.9(10.0)$ & $30.2(12.6)$ & $30.3(16.5)$ & $25.5(12.3)$ & $24.2(13.1)$ & $29.4(14.1)$ \\
\hline \multicolumn{7}{|l|}{ Diabetes type ${ }^{b}$} \\
\hline n (\%) Type I & $217(61.3)$ & $210(60.0)$ & $180(5 \mid .4)$ & $182(52.0)$ & $259(74.0)$ & $\mathrm{I}, 048(59.7)$ \\
\hline n (\%) Type 2 & I 37 (38.7) & $140(40.0)$ & $170(48.6)$ & $168(48.0)$ & $91(26.0)$ & $706(40.3)$ \\
\hline \multicolumn{7}{|l|}{ Age first took insulin ${ }^{b}$} \\
\hline Mean (SD) & $38.7(11.8)$ & $31.8(13.6)$ & $34.0(19.0)$ & $27.0(13.8)$ & $26.2(14.6)$ & $31.6(15.4)$ \\
\hline \multicolumn{7}{|l|}{ Insulin method ${ }^{b}$} \\
\hline n (\%) Needle/syringe & $102(28.8)$ & $54(15.4)$ & $177(50.6)$ & I0I (28.9) & $105(30.0)$ & $539(30.7)$ \\
\hline n (\%) Prefilled/durable pen & 252 (7I.2) & $296(84.6)$ & $173(49.4)$ & $249(71.1)$ & $245(70.0)$ & $\mathrm{I}, 2 \mathrm{I} 5(69.3)$ \\
\hline \multicolumn{7}{|l|}{ Oral medications $\mathrm{s}^{\mathrm{b}}$} \\
\hline n (\%) Yes & $198(55.9)$ & $87(24.9)$ & $142(40.6)$ & $102(29.1)$ & $102(29.1)$ & $631(36.0)$ \\
\hline \multicolumn{7}{|l|}{ Times BG monitored per day ${ }^{b}$} \\
\hline Mean (SD) & $\mathrm{I} .8(1.2)$ & $2.6(2.2)$ & $3.0(1.8)$ & $2.6(1.7)$ & $3.1(2.1)$ & $2.6(1.9)$ \\
\hline \multicolumn{7}{|l|}{ How well controlled ${ }^{\mathrm{b}}$} \\
\hline n (\%) Well/very well & $177(50.0)$ & $275(78.6)$ & $123(35.1)$ & $23 I(66.0)$ & $179(5 \mid .1)$ & $985(56.2)$ \\
\hline \multicolumn{7}{|l|}{ Health status ${ }^{\mathrm{b}}$} \\
\hline n (\%) Good/very good/excellent & $193(54.5)$ & $239(68.3)$ & $2 \mid 4(6 \mid .1)$ & $289(82.6)$ & $253(72.3)$ & $\mathrm{I}, \mathrm{I} 88(67.7)$ \\
\hline \multicolumn{7}{|l|}{ Memory and insulin } \\
\hline \multicolumn{7}{|l|}{ Missed an insulin dose (UF) $)^{b, c}$} \\
\hline n (\%) & $264(74.6)$ & $259(74.0)$ & $225(64.3)$ & $233(66.6)$ & $212(60.6)$ & $\mathrm{I}, 193(68.0)$ \\
\hline \multicolumn{7}{|c|}{ Questioned whether dose taken $(\mathrm{QT})^{\mathrm{b}, \mathrm{d}}$} \\
\hline n (\%) & $294(83.1)$ & $287(82.0)$ & $252(72.0)$ & $297(84.9)$ & $267(76.3)$ & I,397 (79.6) \\
\hline \multicolumn{7}{|c|}{ Questioned dose amount taken (QD) } \\
\hline n (\%) & $233(65.8)$ & $239(68.3)$ & $133(38.0)$ & $199(56.9)$ & I $56(44.6)$ & $960(54.7)$ \\
\hline
\end{tabular}

Notes: Chi-square/ANOVA tests indicate significant differences by country. ${ }^{a} P<0.01$; ${ }^{b} P<0.00$ I; c"You were sure you missed (did not take) an insulin dose (within the last month)"; "you did not question whether or not you missed your dose. This was not deliberate or intentional and may be because you didn't have your insulin or pen/needles/vial with you or they were not available"; "'You questioned yourself, or were unsure, about whether or not you took an insulin dose"; " "You questioned yourself, or were unsure, about how much insulin you took".

Abbreviations: ANOVA, one-way analysis of variance; BG, blood glucose; UF, unintentionally forgetting; QT, questioning whether or not one took dose; QD, questioning how much was taken; SD, standard deviation.

\section{Patient experience of memory problems}

When patients experienced an MP, they had similar concerns across all types of MPs, with the difference that patients had the highest concerns for UF, followed by QT and QD.
Respondents reported that their greatest concern was having high blood glucose readings (hyperglycemia) as a result of nonadherence (UF, 76.3\%; QT, 66.5\%; QD, 60.2\%). Patients also reported being concerned about future diabetes complications 


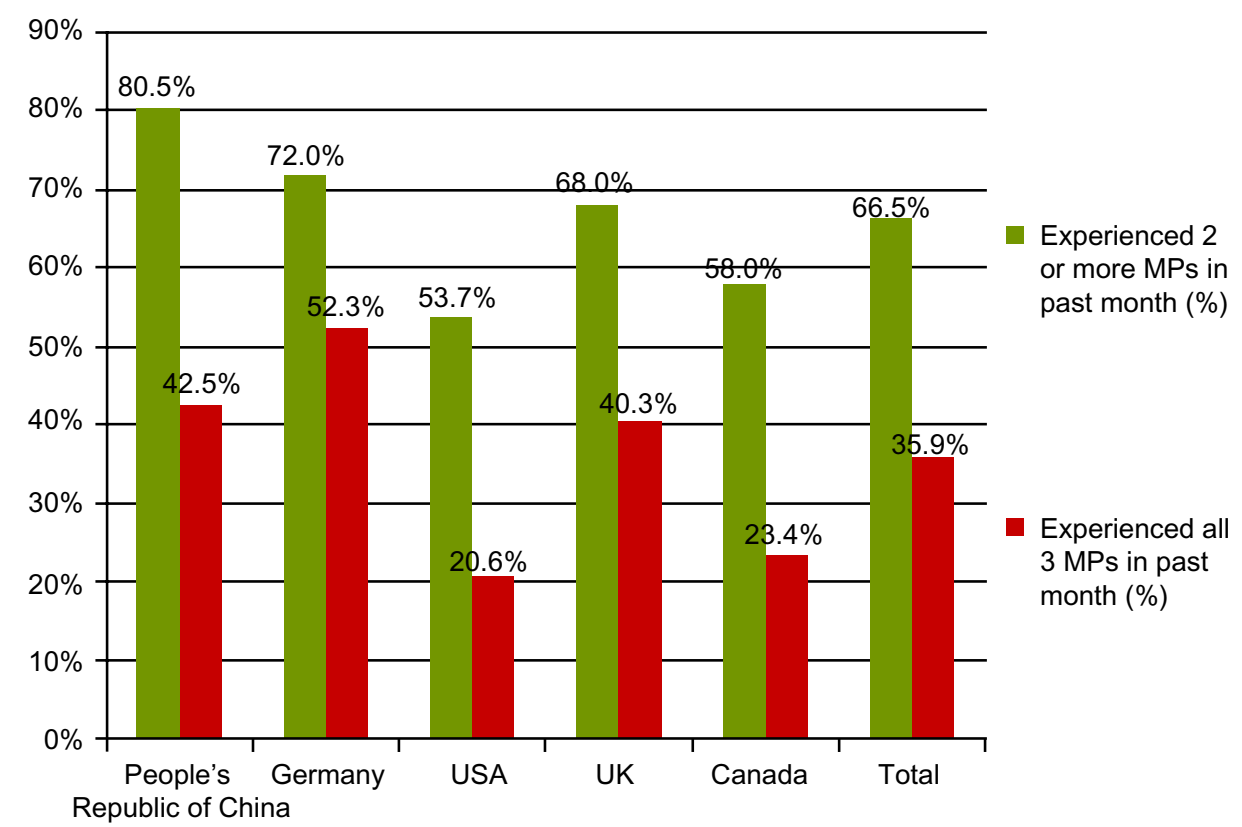

Figure I Incidence of memory problems (MPs) in past month.

as a result of their MP (UF, 64.5\%; QT, 54.0\%; QD, 54.4\%). Significant country differences in respondent concerns were evident. Table 4 shows the most prevalent concerns patients had regarding MPs for the total sample and by country.
The degree of feeling confident about what to do when nonadherent due to an MP was also similar across all three types of MP, with about one third of respondents reporting that they felt "very" or "extremely" confident in knowing what

Table 3 Frequency of memory problems

\begin{tabular}{|c|c|c|c|c|c|c|}
\hline & $\begin{array}{l}\text { People's Republic } \\
\text { of China }\end{array}$ & Germany & USA & UK & Canada & Total \\
\hline \multicolumn{7}{|l|}{ Unintentionally forgetting } \\
\hline Never & $0.4 \%$ & $7.7 \%$ & $4.4 \%$ & $8.2 \%$ & $5.7 \%$ & $5.2 \%$ \\
\hline Very rarely (just a few times a year) & $29.5 \%$ & $23.6 \%$ & $32.4 \%$ & $32.6 \%$ & $43.4 \%$ & $31.9 \%$ \\
\hline Rarely (less than once a month) & $26.5 \%$ & $44.8 \%$ & $21.8 \%$ & $33.0 \%$ & $20.3 \%$ & $29.8 \%$ \\
\hline Sometimes (at least once a month) & $38.3 \%$ & $18.5 \%$ & $25.4 \%$ & $18.0 \%$ & $18.9 \%$ & $24.1 \%$ \\
\hline Often (at least 2-3 times a month) & $4.9 \%$ & $3.1 \%$ & $11.6 \%$ & $5.6 \%$ & $8.0 \%$ & $6.5 \%$ \\
\hline Very often (at least once a week) & $0.4 \%$ & $2.3 \%$ & $4.4 \%$ & $2.6 \%$ & $3.8 \%$ & $2.6 \%$ \\
\hline Weighted average UF events per year & 8.2 & 7.3 & 10.1 & 7.5 & 8.3 & 8.2 \\
\hline \multicolumn{7}{|l|}{ Questioning whether or not took dose } \\
\hline Never & $3.4 \%$ & $6.6 \%$ & $7.5 \%$ & $6.1 \%$ & $7.5 \%$ & $6.2 \%$ \\
\hline Very rarely (just a few times a year) & $32.7 \%$ & $23.0 \%$ & $32.9 \%$ & $37.4 \%$ & $37.5 \%$ & $32.6 \%$ \\
\hline Rarely (less than once a month) & $30.3 \%$ & $44.3 \%$ & $26.6 \%$ & $30.3 \%$ & $25.5 \%$ & $31.6 \%$ \\
\hline Sometimes (at least once a month) & $31.0 \%$ & $17.8 \%$ & $25.0 \%$ & $18.9 \%$ & $20.2 \%$ & $22.5 \%$ \\
\hline Often (at least $2-3$ times a month) & $2.4 \%$ & $7.3 \%$ & $6.0 \%$ & $6.1 \%$ & $5.2 \%$ & $5.4 \%$ \\
\hline Very often (at least once a week) & $0.3 \%$ & $1.0 \%$ & $2.0 \%$ & $1.3 \%$ & $4.1 \%$ & $1.7 \%$ \\
\hline Weighted average QT events per year ${ }^{\mathrm{a}}$ & 6.9 & 7.5 & 7.7 & 7.0 & 8.1 & 7.4 \\
\hline \multicolumn{7}{|l|}{ Questioning how much was taken } \\
\hline Never & $6.9 \%$ & $14.6 \%$ & $20.3 \%$ & $16.1 \%$ & $22.4 \%$ & $15.1 \%$ \\
\hline Very rarely (just a few times a year) & $28.3 \%$ & $14.6 \%$ & $29.3 \%$ & $27.1 \%$ & $36.5 \%$ & $26.1 \%$ \\
\hline Rarely (less than once a month) & $33.0 \%$ & $48.5 \%$ & $27.8 \%$ & $36.2 \%$ & $20.5 \%$ & $30.7 \%$ \\
\hline Sometimes (at least once a month) & $27.9 \%$ & $15.1 \%$ & $17.3 \%$ & $16.6 \%$ & $16.7 \%$ & $19.1 \%$ \\
\hline Often (at least $2-3$ times a month) & $2.6 \%$ & $6.7 \%$ & $5.3 \%$ & $3.0 \%$ & $3.8 \%$ & $4.3 \%$ \\
\hline Very often (at least once a week) & $1.3 \%$ & $0.4 \%$ & $0.0 \%$ & $1.0 \%$ & $0.0 \%$ & $1.0 \%$ \\
\hline Weighted average QD events per year ${ }^{\mathrm{a}}$ & 7.2 & 6.8 & 5.6 & 5.9 & 4.9 & 6.2 \\
\hline
\end{tabular}

Notes: ${ }^{a}$ Weighted average is calculated under the following assumptions: never $=0$ times per year; very rarely (just a few times a year) $=2$ times per year; rarely (less than once a month) $=6$ times per year; sometimes (at least once a month) $=12$ times per year; often (at least 2-3 times a month) $=24$ times per year; very often (at least once a week) $=52$ times per year. 
Table 4 Top concerns regarding memory problems

\begin{tabular}{|c|c|c|c|c|c|c|}
\hline & $\begin{array}{l}\text { People's Republic } \\
\text { of China }\end{array}$ & Germany & USA & UK & Canada & Total \\
\hline \multicolumn{7}{|l|}{ Unintentionally forgetting ${ }^{\mathrm{a}}$} \\
\hline Having high blood sugar readings (hyperglycemia) $P<0.00$ I & $84.4 \%$ & $88.7 \%$ & $74.9 \%$ & $57.0 \%$ & $73.0 \%$ & $76.3 \%$ \\
\hline Future diabetes complications $P<0.00 \mathrm{I}$ & $56.3 \%$ & $80.3 \%$ & $63.7 \%$ & $64.0 \%$ & $57.5 \%$ & $64.5 \%$ \\
\hline Causing family members to worry $P<0.00 \mathrm{I}$ & $63.5 \%$ & $28.9 \%$ & $37.2 \%$ & $20.1 \%$ & $42.0 \%$ & $39.2 \%$ \\
\hline \multicolumn{7}{|l|}{ Questioning whether or not took dose } \\
\hline Having high blood sugar readings (hyperglycemia) $P<0.00 \mathrm{I}$ & $71.8 \%$ & $86.6 \%$ & $70.8 \%$ & $43.4 \%$ & $60.7 \%$ & $66.5 \%$ \\
\hline Having low blood sugar readings (hypoglycemia) $P<0.001$ & $15.8 \%$ & $10.8 \%$ & $29.2 \%$ & $44.8 \%$ & $31.2 \%$ & $26.2 \%$ \\
\hline Future diabetes complications $P<0.00 \mathrm{I}$ & $49.6 \%$ & $73.1 \%$ & $56.2 \%$ & $42.3 \%$ & $49.4 \%$ & $54.0 \%$ \\
\hline Causing family members to worry $P<0.00$ I & $57.7 \%$ & $23.5 \%$ & $29.6 \%$ & $20.8 \%$ & $41.7 \%$ & $34.9 \%$ \\
\hline \multicolumn{7}{|l|}{ Questioning how much was taken ${ }^{\mathrm{a}}$} \\
\hline Having high blood sugar readings (hyperglycemia) $P<0.00$ I & $66.4 \%$ & $85.8 \%$ & $57.5 \%$ & $28.1 \%$ & $52.9 \%$ & $60.2 \%$ \\
\hline Having low blood sugar readings (hypoglycemia) $P<0.00$ I & $23.0 \%$ & $12.3 \%$ & $56.6 \%$ & $71.9 \%$ & $34.7 \%$ & $36.4 \%$ \\
\hline Future diabetes complications $P<0.00 \mathrm{I}$ & $48.4 \%$ & $81.9 \%$ & $55.7 \%$ & $30.5 \%$ & $50.4 \%$ & $54.4 \%$ \\
\hline Causing family members to worry $P<0.001$ & $54.4 \%$ & $25.5 \%$ & $29.2 \%$ & $13.2 \%$ & $33.1 \%$ & $32.3 \%$ \\
\hline
\end{tabular}

Note: auestion stem: "What are you concerned about as a result of...".

to do when experiencing an MP (UF, 34.0\%; QT, 35.9\%; QD, $33.3 \%$ ). Table 5 shows the findings for feelings of confidence for the total sample and by country.

The three most common ways respondents realized that they had an MP were "I just didn't feel right or like myself" (UF, 47.8\%; QT, 51.0\%; QD, 63.2\%), followed by having a high blood glucose test result (UF, 38.8\%; QT, 31.1\%; QD, 35.8\%) or having symptoms due to high blood glucose levels (UF, 29.4\%; QT, 22.8\%; QD, 21.3\%). For all three MPs, being busy was the most common reason given for the MP (UF, 28.5\%; QT, 36.4\%; QD, 33.9\%), followed by being tired (UF, 27.9\%; QT, 33.8\%; QD, 21.9\%) and a major disruption (UF, 25.9\%; QT, 15.6\%; QD, 17.3\%). Significant differences in reasons given for the MP were evident across countries. Figure 2 shows reasons for QT for the total sample and by country.

Respondents most frequently reported being at work (UF, 20.0\%; QT, 15.3\%; QD, 16.8\%), waking in the morning
(UF, 25.8\%; QT, 21.0\%; QD, 15.2\%), relaxing (UF, 18.5\%; QT, 23.5\%; QD, 24.6\%), or doing household jobs/running errands (UF, 12.7\%; QT, 16.2\%; QD, 18.5\%) when their MP occurred. Significant country differences were apparent for each MP, but differences varied across types of MP. The majority of respondents (QT, 69.6\%; QD, 80.4\%) reported using a strategy or visual cue (such as putting an insulin pen in a particular place) to find out whether the appropriate insulin dose or dose amount was taken when experiencing QT or QD. More specifically, most respondents indicated that they used a strategy or visual cue and knew for sure that the appropriate dose was taken (QT, 32.0\%; QD, 38.9\%), followed by using a strategy or visual cue and knowing that the appropriate dose was not taken (QT, 23.1\%; QD, 26.5\%), using a strategy or visual cue and still questioning (QT, 14.5\%; QD, 15.0\%), and not using a strategy or visual cue (QT, 30.4\%; QD, 19.6\%).

Table 5 Confidence when experiencing memory problems

\begin{tabular}{|c|c|c|c|c|c|c|}
\hline Miss an insulin dose? (UF) & $\begin{array}{l}\text { People's Republic } \\
\text { of China }\end{array}$ & Germany & USA & UK & Canada & $\begin{array}{l}\text { Total } \\
(n=I,|3|)\end{array}$ \\
\hline Not at all/a little & $35.0 \%$ & $8.8 \%$ & $28.4 \%$ & $41.6 \%$ & $34.0 \%$ & $29.3 \%$ \\
\hline Somewhat & $54.0 \%$ & $19.2 \%$ & $34.9 \%$ & $38.3 \%$ & $35.5 \%$ & $36.7 \%$ \\
\hline Very/extremely & $11.0 \%$ & $72.0 \%$ & $36.7 \%$ & $20.1 \%$ & $30.5 \%$ & $34.0 \%$ \\
\hline $\begin{array}{l}\text { Question whether or not you } \\
\text { took your insulin dose? }\end{array}$ & $\begin{array}{l}\text { People's Republic } \\
\text { of China }\end{array}$ & Germany & USA & UK & Canada & $\begin{array}{l}\text { Total } \\
(n=I,|3|)\end{array}$ \\
\hline Not at all/a little & $31.0 \%$ & $11.6 \%$ & $22.7 \%$ & $30.8 \%$ & $25.5 \%$ & $24.5 \%$ \\
\hline Somewhat & $58.4 \%$ & $18.3 \%$ & $39.1 \%$ & $40.5 \%$ & $40.5 \%$ & $39.6 \%$ \\
\hline Very/extremely & $10.6 \%$ & $70.1 \%$ & $38.2 \%$ & $28.7 \%$ & $34.0 \%$ & $35.9 \%$ \\
\hline $\begin{array}{l}\text { Are unsure about how much } \\
\text { insulin you took? }\end{array}$ & $\begin{array}{l}\text { People's Republic } \\
\text { of China }\end{array}$ & Germany & USA & UK & Canada & $\begin{array}{l}\text { Total } \\
(n=8 \mid 5)\end{array}$ \\
\hline Not at all/a little & $30.0 \%$ & $7.4 \%$ & $34.0 \%$ & $36.5 \%$ & $28.1 \%$ & $25.9 \%$ \\
\hline Somewhat & $57.1 \%$ & $19.6 \%$ & $35.8 \%$ & $50.3 \%$ & $38.8 \%$ & $40.9 \%$ \\
\hline Very/extremely & $12.9 \%$ & $73.0 \%$ & $30.2 \%$ & $13.2 \%$ & $33.1 \%$ & $33.2 \%$ \\
\hline
\end{tabular}

Note: aQuestion stem: "How confident are you that you know what to do when you...".

Abbreviation: UF, unintentionally forgetting. 


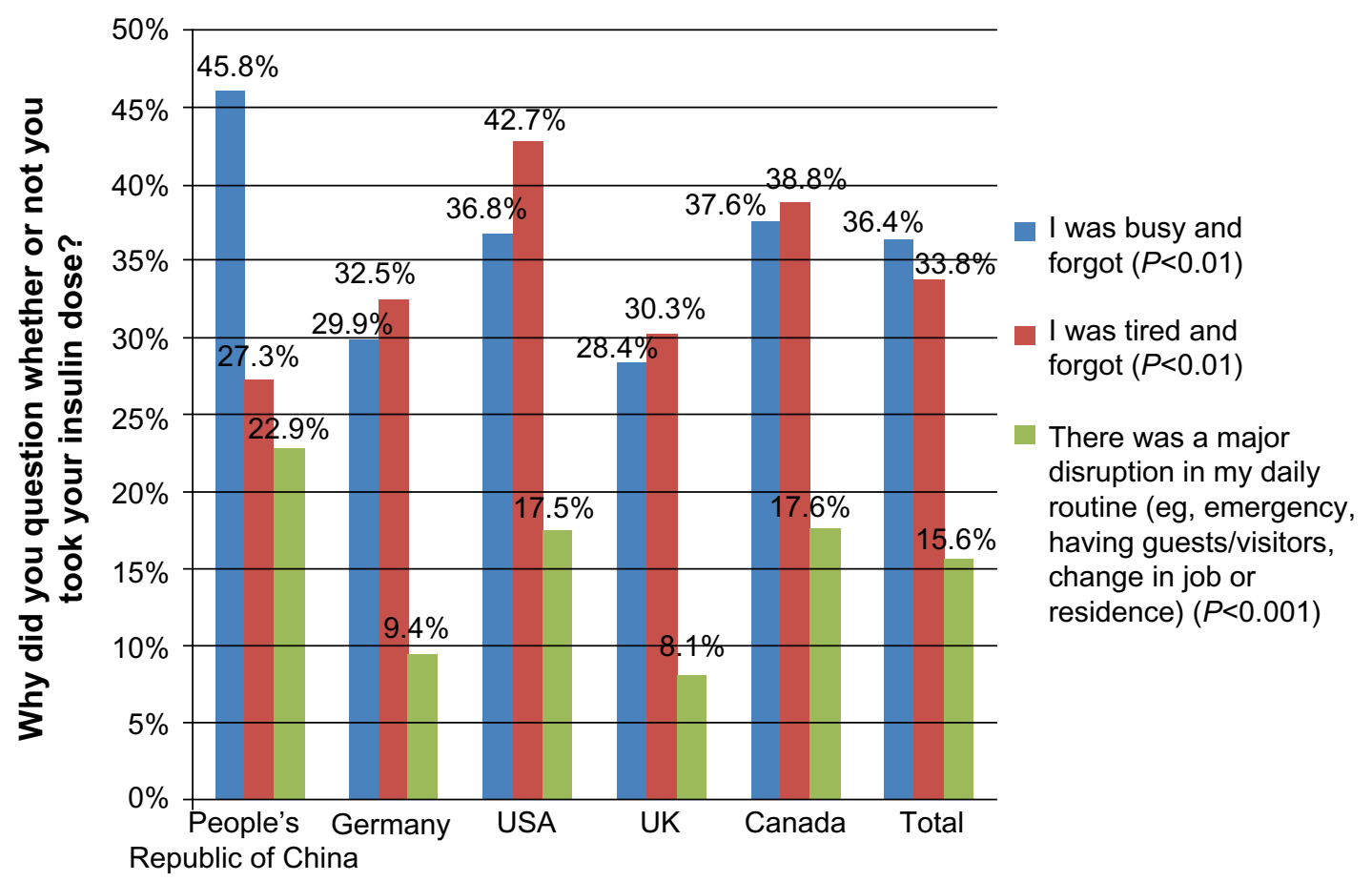

Figure 2 Top three reasons for questioning whether or not took dose. Note: Responses not mutually exclusive.

\section{Patient concerns about memory problems}

Based on a 10-point scale ( 0 , not at all worried; 10 , extremely worried), respondents expressed moderate worry about double dosing, hypoglycemia, and hyperglycemia when considering which corrective action to take following their MP. Respondents who experienced QT had an average rating of 5.8 while those who experienced a QD had an average rating of 6.1 for concerns about double dosing when making a corrective action decision. Respondent concerns about low blood glucose, ie, hypoglycemia (UF, 5.6; QT, 5.5; QD, 5.9) and high blood glucose, ie, hyperglycemia (UF, 6.6; QT, 6.3; QD, 6.6) when considering corrective actions following an MP were also moderate.

\section{Corrective actions taken following memory problems}

Respondents reported that after experiencing an MP, they took corrective action by measuring their blood glucose level to determine their next action, taking an insulin dose without measuring blood glucose, or taking no action (skipping a dose and waiting for the next scheduled dose). For UF, the majority of respondents reported measuring their blood glucose and taking an insulin dose based on the reading (45.8\%), followed by skipping a dose (27.4\%) and taking an insulin dose without measuring blood glucose (26.7\%). For QT, the majority of respondents responded by skipping a dose (39.6\%), followed by measuring their blood glucose and taking insulin based on the reading (38.4\%) and taking an insulin dose without measuring blood glucose (22.1\%). For QD, the majority of respondents measured their blood glucose and took insulin based on the reading $(41.3 \%)$, followed by skipping a dose (32.7\%) and taking an insulin dose without measuring blood glucose (26.0\%). For both UF and QD, patients who measured their blood glucose following the MP were significantly more likely to report experiencing hyperglycemia following the MP. Table 6 shows the corrective actions taken after having an MP for the total sample and by country.

\section{Consequences of memory problems Recovery time}

For each type of MP, about half of respondents reported experiencing high blood glucose symptoms (hyperglycemia) as a consequence of their MP (UF, 58.4\%; QT, 48.7\%; QD, $46.4 \%$ ). For those experiencing high blood sugar symptoms, recovery times for UF were longer than those for QT and QD, as shown in Table 7. On average, respondents reported that it took 7.7 hours for their blood glucose to return to the normal range following UF (QT, 1.2 hours; QD, 1.0 hour). Respondents reported that it took an average of 7.2 hours for high blood glucose symptoms to resolve following UF (QT, 1.0 hour; QD, 1.1 hours). For UF, respondents also reported that it took 8.4 hours to return to their usual level 
Table 6 Corrective actions following memory problems

\begin{tabular}{|c|c|c|c|c|c|c|}
\hline Unintentionally forgetting & $\begin{array}{l}\text { People's } \\
\text { Republic of China } \\
n=230\end{array}$ & $\begin{array}{l}\text { Germany } \\
n=104\end{array}$ & $\begin{array}{l}\text { USA } \\
n=|8|\end{array}$ & $\begin{array}{l}\text { UK } \\
n=190\end{array}$ & $\begin{array}{l}\text { Canada } \\
n=\mid 55\end{array}$ & $\begin{array}{l}\text { Total } \\
n=860\end{array}$ \\
\hline $\begin{array}{l}\text { Tested my blood sugar and took a dose of insulin } \\
\text { based on the reading (\%) }\end{array}$ & 61.3 & 46.2 & 37.6 & 41.6 & 37.4 & 45.8 \\
\hline $\begin{array}{l}\text { Took a dose of insulin without testing my blood } \\
\text { sugar before taking the dose (\%) }\end{array}$ & 17.0 & 26.0 & 23.2 & 44.7 & 23.9 & 26.7 \\
\hline $\begin{array}{l}\text { Waited (skipped dose) and took next regularly } \\
\text { scheduled dose of insulin (did nothing) (\%) }\end{array}$ & 21.7 & 27.9 & 39.2 & 13.7 & 38.7 & 27.4 \\
\hline Questioning whether or not took dose & $\begin{array}{l}\text { People's } \\
\text { Republic of China } \\
n=96\end{array}$ & $\begin{array}{l}\text { Germany } \\
n=56\end{array}$ & $\begin{array}{l}\text { USA } \\
n=86\end{array}$ & $\begin{array}{l}\text { UK } \\
n=86\end{array}$ & $\begin{array}{l}\text { Canada } \\
n=93\end{array}$ & $\begin{array}{l}\text { Total } \\
n=417\end{array}$ \\
\hline $\begin{array}{l}\text { Tested my blood sugar and took a dose of insulin } \\
\text { based on the reading (\%) }\end{array}$ & 55.2 & 44.6 & 36.0 & 36.0 & 21.5 & 38.4 \\
\hline $\begin{array}{l}\text { Took a dose of insulin without testing my blood } \\
\text { sugar before taking the dose (\%) }\end{array}$ & 12.5 & 14.3 & 23.3 & 31.4 & 26.9 & 22.1 \\
\hline $\begin{array}{l}\text { Waited (skipped dose) and took next regularly } \\
\text { scheduled dose of insulin (did nothing) (\%) }\end{array}$ & 32.3 & $4 I .1$ & 40.7 & 32.6 & 51.6 & 39.6 \\
\hline Questioning how much was taken & $\begin{array}{l}\text { People's } \\
\text { Republic of China } \\
\text { n=74 }\end{array}$ & $\begin{array}{l}\text { Germany } \\
n=29\end{array}$ & $\begin{array}{l}\text { USA } \\
n=33\end{array}$ & $\begin{array}{l}\text { UK } \\
n=50\end{array}$ & $\begin{array}{l}\text { Canada } \\
n=49\end{array}$ & $\begin{array}{l}\text { Total } \\
n=235\end{array}$ \\
\hline $\begin{array}{l}\text { Tested my blood sugar and took a dose of insulin } \\
\text { based on the reading (\%) }\end{array}$ & 64.8 & 34.5 & 30.3 & 24.0 & 34.7 & 41.3 \\
\hline $\begin{array}{l}\text { Took a dose of insulin without testing my blood } \\
\text { sugar before taking the dose (\%) }\end{array}$ & 12.2 & 17.2 & 18.2 & 50.0 & 32.7 & 26.0 \\
\hline $\begin{array}{l}\text { Waited (skipped dose) and took next regularly } \\
\text { scheduled dose of insulin (did nothing) (\%) }\end{array}$ & 23.0 & 48.3 & 51.5 & 26.0 & 32.6 & 32.7 \\
\hline
\end{tabular}

of physical functioning (QT, 1.4 hours; QD, 1.1 hours) and 9.6 hours to return to their usual level of emotional functioning (QT, 1.5 hours; QD, 1.2 hours). Table 7 shows the recovery times after an MP for the total sample and by country.

\section{Impact on functioning and well-being}

Respondents consistently reported that MPs had a moderate negative impact on both physical and emotional functioning outside of work. On a 10-point scale (0, no impact; 10 , extremely negative impact), respondents reported that MPs had a moderate negative impact on physical functioning the following day (UF, 5.6; QT, 5.3; QD, 5.8), which included performing one's normal daily activities at one's usual level (outside of work). Respondents also reported that MPs had a moderate negative impact on emotional functioning the following day (UF, 5.8; QT, 5.5; QD, 6.2), which again includes performing one's normal daily activities at one's usual level (outside of work).

\section{Impact on diabetes management}

Respondents conducted, on average, an additional 1.4 extra blood glucose measurements following a UF, while those who experienced a QT or QD measured their blood glucose less than one extra time, on average, in the 24 hours after the MP (QT, 0.6; QD, 0.7, shown in Table 8). Those experiencing UF conducted an average of 4.9 extra blood glucose measurements in the 7 days following the MP (QT, 1.8; QD, 2.4). Table 8 shows the impact of MPs on diabetes management for the total sample and by country.

\section{Impact on work}

Respondents were asked to indicate the impact MPs had on their functioning using numbers based on a 10-point scale ( 0 , no impact; 10 , extremely negative impact). As shown in Table 9, patients reported that MPs had a moderate negative impact on physical functioning at work (UF, 6.1; QT, 5.6; QD, 6.0). Likewise, respondents indicated that MPs had a moderate negative impact on emotional functioning at work (UF, 6.4; QT, 5.8; QD, 6.5). Table 9 shows the impact of MPs on functioning while at work for the total sample and by country.

Close to one fifth of respondents reported that they were late to work (UF, $18.9 \%$; QT, $17.3 \%$; QD, $19.9 \%$ ) or left work early (UF, 20.6\%; QT, 14.8\%; QD, 21.5\%) as a result of their MP. Additionally, for all MPs, close to $10 \%$ 
Table 7 Mean number of hours to recovery following memory problems

\begin{tabular}{|c|c|c|c|c|c|c|}
\hline & $\begin{array}{l}\text { People's } \\
\text { Republic of } \\
\text { China }\end{array}$ & Germany & USA & UK & Canada & Total \\
\hline \multicolumn{7}{|l|}{ Unintentionally forgetting } \\
\hline $\begin{array}{l}\text { This last time, how long did it take for you to get your } \\
\text { high blood sugar levels back into a normal range? }\end{array}$ & $8.3(n=225)$ & $6.4(n=101)$ & $8.6(n=165)$ & $5.8(n=178)$ & $8.7(n=147)$ & $7.7(n=8 \mid 6)$ \\
\hline $\begin{array}{l}\text { How long did it take for all of your high blood sugar } \\
\text { symptoms to go away? }\end{array}$ & $8.7(n=2 \mid 8)$ & $6.2(n=100)$ & $7.3(n=170)$ & $6.0(n=183)$ & $6.9(n=144)$ & $7.2(n=8 \mid 5)$ \\
\hline $\begin{array}{l}\text { How long did it take until you felt you were physically } \\
\text { functioning again at your usual normal level? }\end{array}$ & $9.5(n=2 \mid 2)$ & $6.2(n=102)$ & $9.8(n=172)$ & $7.0(n=182)$ & $8.7(n=148)$ & $8.4(n=8 \mid 6)$ \\
\hline $\begin{array}{l}\text { How long did it take until you felt you were emotionally } \\
\text { functioning again at your usual normal level? }\end{array}$ & $1 \mathrm{I} .6(\mathrm{n}=2 \mathrm{II})$ & $9.8(n=102)$ & $9.3(n=|7|)$ & $8.2(n=184)$ & $8.8(n=148)$ & $9.6(n=8 \mid 6)$ \\
\hline \multicolumn{7}{|l|}{ Questioning whether or not took dose } \\
\hline $\begin{array}{l}\text { This last time, how long did it take for you to get your } \\
\text { high blood sugar levels back into a normal range? }\end{array}$ & $1.2(n=209)$ & $1.5(n=115)$ & $1.3(n=159)$ & $0.7(n=203)$ & $1.7(n=161)$ & $1.2(n=847)$ \\
\hline $\begin{array}{l}\text { How long did it take for all of your high blood sugar } \\
\text { symptoms to go away? }\end{array}$ & $1.2(n=205)$ & I.I $(n=115)$ & $0.9(n=162)$ & $0.5(n=204)$ & $1.4(n=161)$ & $1.0(n=847)$ \\
\hline $\begin{array}{l}\text { How long did it take until you felt you were physically } \\
\text { functioning again at your usual normal level? }\end{array}$ & $1.3(n=205)$ & $1.3(n=114)$ & $1.7(n=163)$ & $0.8(n=205)$ & $1.9(n=163)$ & $1.4(n=850)$ \\
\hline $\begin{array}{l}\text { How long did it take until you felt you were emotionally } \\
\text { functioning again at your usual normal level? }\end{array}$ & $1.8(n=207)$ & $1.0(n=113)$ & $1.4(n=161)$ & $0.8(n=205)$ & $2.3(n=164)$ & $1.5(n=850)$ \\
\hline \multicolumn{7}{|l|}{ Questioning how much was taken } \\
\hline $\begin{array}{l}\text { This last time, how long did it take for you to get your } \\
\text { high blood sugar levels back into a normal range? }\end{array}$ & $0.7(n=|5|)$ & $1.3(n=56)$ & $1.5(n=73)$ & $0.7(n=|4|)$ & $1.9(n=79)$ & $1.0(n=500)$ \\
\hline $\begin{array}{l}\text { How long did it take for all of your high blood sugar } \\
\text { symptoms to go away? }\end{array}$ & $0.8(n=|5|)$ & $1.6(n=56)$ & $1.6(n=74)$ & $0.5(n=|4|)$ & $1.8(n=76)$ & I.I $(n=498)$ \\
\hline $\begin{array}{l}\text { How long did it take until you felt you were physically } \\
\text { functioning again at your usual normal level? }\end{array}$ & $0.8(n=|5|)$ & $0.9(n=55)$ & I.I $(n=73)$ & $0.5(n=140)$ & $2.7(n=79)$ & I.I $(n=498)$ \\
\hline $\begin{array}{l}\text { How long did it take until you felt you were emotionally } \\
\text { functioning again at your usual normal level? }\end{array}$ & I.I $(n=152)$ & $0.8(n=55)$ & $1.4(n=74)$ & $0.5(n=140)$ & $2.8(n=77)$ & $1.2(n=498)$ \\
\hline
\end{tabular}

Note: Data were trimmed by $5 \%$ at the upper level for each variable to exclude probable reporting errors.

reported that they missed a full day of work as a result of the MP (UF, 12.0\%; QT, 7.6\%; QD, 12.7\%), and slightly more indicated that they missed a meeting or work appointment due to the MP (UF, 21.8\%; QT, 14.8\%; QD, 18.2\%). Based on the frequency of MPs per patient per year (Table 3), this roughly corresponds to patients going late to work 1.5 times per year (UF, 1.5; QT, 1.3; QD, 1.2), leaving work early approximately 1.5 times per year (UF, 1.7; QT, 1.1; QD, 1.3), and missing on average one full day of work per year (UF, 1.0; QT, 0.6; QD, 0.8) as a result of MPs. On average, patients missed about 1.5 meetings or work appointments due to the MP (UF, 1.8; QT, 1.1; QD, 1.1). Table 10 shows the impact of MPs on ability to function at work for the total sample and by country.

For both UF and QT, measuring blood glucose was associated with a significantly greater likelihood of missing work time, including going in late to work, leaving work early, missing work-related appointments or meetings, and missing a full day of work. Among those who measured blood glucose following a UF, $45.1 \%$ reported missing work compared with $32.4 \%$ of those who do not measure blood glucose. Likewise, among those who measured blood glucose following a QT, 39.1\% reported missed work, compared with 17.5\% of those who did not measure blood glucose. Although QD showed a similar pattern, the differences were not statistically significant. Table 10 shows the impact of MPs on missed work time for the total sample and by country.

\section{Contact with health care professionals}

For all three MPs, a number of patients reported visiting a physician or other health care professional because of their MP or how they felt as a result of the MP (UF, 20.8\%; QT, $14.6 \%$; QD, 21.3\%). Similarly, a number of patients also reported calling or emailing a physician or health care professional because of their MP (UF, 23.4\%; QT, 16.1\%; QD, 24.7\%). Table 11 shows the impact of MPs on contacts with health care professionals for the total sample and by country.

\section{Influence of age, diabetes type, duration of diabetes, and insulin regime on MPs} Unintentional forgetting

For UF, the results showed some significant variation by age, type of diabetes, and duration of disease, as measured by years with a diagnosis of diabetes. Age was significantly associated 
Table 8 Additional blood glucose measurements following memory problems

\begin{tabular}{|c|c|c|c|c|c|c|}
\hline Unintentionally forgetting & $\begin{array}{l}\text { People's Republic } \\
\text { of China }\end{array}$ & Germany & USA & UK & Canada & $\begin{array}{l}\text { Total } \\
(n=860)\end{array}$ \\
\hline Average extra BG strips used (I day) & 1.5 & 1.5 & 1.1 & 1.4 & 1.5 & 1.4 \\
\hline Average extra BG strips used (7 days) & 4.4 & 4.4 & 3.7 & 6.8 & 5.0 & 4.9 \\
\hline Extra BG strips used per patient per year (I day $)^{\mathrm{a}}$ & 12.2 & 10.7 & II.I & 10.1 & 12.4 & 11.5 \\
\hline Extra BG strips used per patient per year (7 days) ${ }^{a}$ & 35.6 & 32.1 & 37.4 & 51.2 & 41.4 & 40.2 \\
\hline Questioning whether or not took dose & $\begin{array}{l}\text { People's Republic } \\
\text { of China }\end{array}$ & Germany & USA & UK & Canada & $\begin{array}{l}\text { Total } \\
(n=896)\end{array}$ \\
\hline Average extra BG strips used (I day) & 0.6 & 0.7 & 0.7 & 0.5 & 0.6 & 0.6 \\
\hline Average extra BG strips used (7 days) & 1.9 & 1.2 & 2.4 & 1.7 & 1.9 & 1.8 \\
\hline Extra BG strips used per patient per year (I day $)^{\mathrm{a}}$ & 4.4 & 5.0 & 5.4 & 3.2 & 5.2 & 4.4 \\
\hline Extra BG strips used per patient per year ( 7 days $)^{a}$ & 13.1 & 8.7 & 18.5 & 12.0 & 15.4 & 13.3 \\
\hline Questioning how much was taken & $\begin{array}{l}\text { People's Republic } \\
\text { of China }\end{array}$ & Germany & USA & UK & Canada & $\begin{array}{l}\text { Total } \\
(n=525)\end{array}$ \\
\hline Average extra BG strips used (I day) & 0.8 & 0.8 & 0.7 & 0.5 & 0.7 & 0.7 \\
\hline Average extra BG strips used (7 days) & 2.5 & 2.1 & 2.4 & 2.6 & 2.5 & 2.4 \\
\hline Extra BG strips used per patient per year (I day $)^{\mathrm{a}}$ & 5.6 & 5.5 & 3.9 & 2.8 & 3.4 & 4.3 \\
\hline Extra BG strips used per patient per year (7 days) ${ }^{\mathrm{a}}$ & 17.7 & 14.2 & 13.4 & 15.6 & 12.1 & 14.9 \\
\hline
\end{tabular}

Note: a Average extra BG strips used multiplied by the number of events per patient per year.

Abbreviation: BG, blood glucose.

with frequency of UF, corrective action taken following UF, missed work time, and health care contact due to UF. Specifically, those who experienced UF either "often/very often" had a slightly higher average age (43.8 years) than those who experienced UF less frequently ("sometimes", 41.5 years; "very rarely/rarely", 40.2 years; "never", 42.3 years, $P<0.05$ ). Additionally, in terms of corrective action taken following UF, those who skipped a dose were significantly older ( 45.8 years) than those who measured blood glucose and took insulin based on a reading (39.1 years) and those who took insulin without measuring blood glucose $(40.5$ years, $P<0.001)$. Moreover, those who missed work time were significantly younger (mean age 36.1 years) than those who did not miss time at work (39.1 years, $P<0.01$ ). Also, those who contacted health care professionals due to an MP were significantly younger (37.4 years) than those who did not contact health care professionals (43.2 years, $P<0.001$ ).

Type of diabetes also had a significant impact on some results for UF, including corrective action and health care contact. Specifically, subjects with T2D were significantly

Table 9 Impact of memory problems on functioning at work and missed work time

\begin{tabular}{|c|c|c|c|c|c|c|}
\hline Unintentionally forgetting & $\begin{array}{l}\text { People's } \\
\text { Republic of China } \\
\mathrm{n}=\mathbf{2 0 3}\end{array}$ & $\begin{array}{l}\text { Germany } \\
n=81\end{array}$ & $\begin{array}{l}\text { USA } \\
n=95\end{array}$ & $\begin{array}{l}\text { UK } \\
n=158\end{array}$ & $\begin{array}{l}\text { Canada } \\
n=100\end{array}$ & $\begin{array}{l}\text { Total } \\
n=637\end{array}$ \\
\hline $\begin{array}{l}\text { On a scale of } 0-10 \text {, impact your ability to function } \\
\text { physically at your usual level at work? Mean (SD) }\end{array}$ & $6.5(2.4)$ & $6.8(2.7)$ & $5.4(3.2)$ & $5.9(2.4)$ & $5.7(2.4)$ & $6.1(2.6)$ \\
\hline $\begin{array}{l}\text { On a scale of } 0-10 \text {, impact your ability to function } \\
\text { emotionally at your usual level at work? Mean (SD) }\end{array}$ & $6.7(2.5)$ & $6.7(2.7)$ & $5.3(3.2)$ & $6.8(2.4)$ & $5.7(2.4)$ & $6.4(2.6)$ \\
\hline Questioning whether or not took dose & $\begin{array}{l}\text { People's } \\
\text { Republic of China } \\
n=85\end{array}$ & $\begin{array}{l}\text { Germany } \\
n=43\end{array}$ & $\begin{array}{l}\text { USA } \\
n=43\end{array}$ & $\begin{array}{l}\text { UK } \\
n=65\end{array}$ & $\begin{array}{l}\text { Canada } \\
n=6 I\end{array}$ & $\begin{array}{l}\text { Total } \\
\mathbf{n}=297\end{array}$ \\
\hline $\begin{array}{l}\text { On a scale of } 0-10 \text {, impact your ability to function } \\
\text { physically at your usual level at work? Mean (SD) }\end{array}$ & $6.7(2.4)$ & $5.2(3.0)$ & $5.4(2.9)$ & $5.4(2.7)$ & $4.6(2.7)$ & $5.6(2.8)$ \\
\hline $\begin{array}{l}\text { On a scale of } 0-10 \text {, impact your ability to function } \\
\text { emotionally at your usual level at work? Mean (SD) }\end{array}$ & $6.8(2.5)$ & $5.2(3.1)$ & $5.3(2.9)$ & $6.1(2.8)$ & $4.8(2.8)$ & $5.8(2.9)$ \\
\hline Questioning how much was taken & $\begin{array}{l}\text { People's } \\
\text { Republic of China } \\
\mathrm{n}=68\end{array}$ & $\begin{array}{l}\text { Germany } \\
n=23\end{array}$ & $\begin{array}{l}\text { USA } \\
n=I 5\end{array}$ & $\begin{array}{l}\text { UK } \\
n=45\end{array}$ & $\begin{array}{l}\text { Canada } \\
\mathrm{n}=38\end{array}$ & $\begin{array}{l}\text { Total } \\
n=189\end{array}$ \\
\hline $\begin{array}{l}\text { On a scale of } 0-10 \text {, impact your ability to function } \\
\text { physically at your usual level at work? Mean (SD) }\end{array}$ & $6.7(2.0)$ & $5.2(2.7)$ & $6.5(2.1)$ & $5.7(2.6)$ & $5.3(2.4)$ & $6.0(2.4)$ \\
\hline $\begin{array}{l}\text { On a scale of } 0-10 \text {, impact your ability to function } \\
\text { emotionally at your usual level at work? Mean (SD) }\end{array}$ & $7.1(2.3)$ & $5.3(2.9)$ & $6.3(2.2)$ & $6.8(2.2)$ & $5.7(2.8)$ & $6.5(2.5)$ \\
\hline
\end{tabular}

Abbreviation: SD, standard deviation. 
Table 10 Impact of memory problems on ability to function at work

\begin{tabular}{|c|c|c|c|c|c|c|}
\hline Unintentionally forgetting & $\begin{array}{l}\text { People's } \\
\text { Republic of China } \\
\mathrm{n}=203\end{array}$ & $\begin{array}{l}\text { Germany } \\
n=81\end{array}$ & $\begin{array}{l}\text { USA } \\
n=95\end{array}$ & $\begin{array}{l}\text { UK } \\
n=I 58\end{array}$ & $\begin{array}{l}\text { Canada } \\
n=100\end{array}$ & $\begin{array}{l}\text { Total } \\
n=637\end{array}$ \\
\hline Did you go late to work? n (\%) & $50(25.0)$ & $8(10.5)$ & II (I3.6) & $33(22.0)$ & $12(12.6)$ & $114(18.9)$ \\
\hline Total minutes, mean (SD) & $63.8(57.8)$ & $226.3(254.5)$ & $73.8(90.7)$ & $123.8(126.4)$ & $161.1(176.1)$ & $103.8(126.7)$ \\
\hline Did you leave work early? n (\%) & $49(24.5)$ & $10(13.2)$ & $10(12.3)$ & $32(21.3)$ & $23(24.2)$ & $124(20.6)$ \\
\hline Total minutes, mean (SD) & $94.5(69.4)$ & $86.5(49.3)$ & $153.9(109.7)$ & $120.0(104.1)$ & $78.3(77.7)$ & $102.2(84.8)$ \\
\hline Did you miss a full day? n (\%) & $23(11.5)$ & $9(11.8)$ & $5(6.2)$ & $22(14.7)$ & $13(13.7)$ & $72(12.0)$ \\
\hline $\begin{array}{l}\text { Did you miss a meeting, a work appointment } \\
\text { or did you not finish a task on time? } n(\%)\end{array}$ & $56(28.0)$ & $10(13.2)$ & $15(18.5)$ & $32(21.3)$ & $18(18.9)$ & $|3|(2 \mid .8)$ \\
\hline $\begin{array}{l}\text { Average numbers of times going late to } \\
\text { work per patient per year }{ }^{\mathrm{a}}\end{array}$ & 2.1 & 0.8 & 1.4 & 1.7 & 1.0 & 1.5 \\
\hline $\begin{array}{l}\text { Average numbers of times leaving work } \\
\text { early per patient per year }\end{array}$ & 2.0 & 1.0 & 1.2 & 1.6 & 2.0 & 1.7 \\
\hline $\begin{array}{l}\text { Average numbers of times missing a full day } \\
\text { per patient per year }{ }^{\mathrm{a}}\end{array}$ & 0.9 & 0.9 & 0.6 & I.I & 1.1 & 1.0 \\
\hline $\begin{array}{l}\text { Average numbers of times miss a meeting, a } \\
\text { work appointment or did not finish a task on } \\
\text { time per patient per year }{ }^{\mathrm{a}}\end{array}$ & 2.3 & 1.0 & 1.9 & 1.6 & 1.6 & 1.8 \\
\hline Questioning whether or not took dose & $\begin{array}{l}\text { People's } \\
\text { Republic of China } \\
n=85\end{array}$ & $\begin{array}{l}\text { Germany } \\
n=43\end{array}$ & $\begin{array}{l}\text { USA } \\
n=43\end{array}$ & $\begin{array}{l}\text { UK } \\
n=65\end{array}$ & $\begin{array}{l}\text { Canada } \\
n=6 I\end{array}$ & $\begin{array}{l}\text { Total } \\
\mathrm{n}=297\end{array}$ \\
\hline Did you go late to work? n (\%) & $26(31.0)$ & $4(10.0)$ & $5(13.2)$ & $6(10.0)$ & $7(12.7)$ & $48(17.3)$ \\
\hline Total minutes, mean (SD) & $54.2(41.0)$ & $228.0(183.6)$ & $94.6(80.7)$ & $198.8(151.6)$ & $107.9(109.8)$ & $98.8(106.8)$ \\
\hline Did you leave work early? n (\%) & $24(28.6)$ & $2(5.0)$ & $5(13.2)$ & $3(5.0)$ & $7(12.7)$ & $4 I(14.8)$ \\
\hline Total minutes, mean (SD) & $80.0(51.6)$ & $120.0(0.0)$ & $163.4(148.4)$ & $290.0(131.8)$ & $127.3(128.1)$ & $115.6(102.1)$ \\
\hline Did you miss a full day? n (\%) & $9(10.7)$ & $2(5.0)$ & $4(10.5)$ & $3(5.0)$ & $3(5.5)$ & $21(7.6)$ \\
\hline $\begin{array}{l}\text { Did you miss a meeting, a work appointment } \\
\text { or did you not finish a task on time? } \mathrm{n}(\%)\end{array}$ & $20(23.8)$ & $4(10.0)$ & $5(13.2)$ & 7 (II.7) & $5(9.1)$ & $4 \mid(\mid 4.8)$ \\
\hline $\begin{array}{l}\text { Average numbers of times going late to } \\
\text { work per patient per year }{ }^{\mathrm{a}}\end{array}$ & 2.1 & 0.8 & 1.0 & 0.7 & 1.0 & 1.3 \\
\hline $\begin{array}{l}\text { Average numbers of times leaving work } \\
\text { early per patient per year }\end{array}$ & 2.0 & 0.4 & 1.0 & 0.4 & 1.0 & $\mathrm{I} . \mathrm{I}$ \\
\hline $\begin{array}{l}\text { Average numbers of times missing a full day } \\
\text { per patient per year }{ }^{\mathrm{a}}\end{array}$ & 0.7 & 0.4 & 0.8 & 0.4 & 0.4 & 0.6 \\
\hline $\begin{array}{l}\text { Average numbers of times miss a meeting, a } \\
\text { work appointment or did not finish a task on } \\
\text { time per patient per year }{ }^{\mathrm{a}}\end{array}$ & 1.6 & 0.8 & 1.0 & 0.8 & 0.7 & I.I \\
\hline Questioning how much was taken & $\begin{array}{l}\text { People's } \\
\text { Republic of China } \\
\mathrm{n}=68\end{array}$ & $\begin{array}{l}\text { Germany } \\
n=23\end{array}$ & $\begin{array}{l}\text { USA } \\
n=15\end{array}$ & $\begin{array}{l}\text { UK } \\
n=45\end{array}$ & $\begin{array}{l}\text { Canada } \\
\mathrm{n}=38\end{array}$ & $\begin{array}{l}\text { Total } \\
n=189\end{array}$ \\
\hline Did you go late to work? n (\%) & $21(31.3)$ & $2(9.5)$ & $2(13.3)$ & $5(11.4)$ & $6(17.6)$ & $36(19.9)$ \\
\hline Total minutes, mean (SD) & $68.9(68.7)$ & $150.0(42.2)$ & $30.0(42.4)$ & $44.8(48.3)$ & $56.5(93.4)$ & $65.8(69.8)$ \\
\hline Did you leave work early? n (\%) & $18(26.9)$ & $4(19.0)$ & $2(13.3)$ & $6(13.6)$ & $9(26.5)$ & $39(21.5)$ \\
\hline Total minutes, mean (SD) & $65.1(46.3)$ & $125.0(75.5)$ & $30.0(42.4)$ & I 57.7 (I20.5) & $51.1(74.4)$ & $80.4(78.3)$ \\
\hline Did you miss a full day? n (\%) & $9(13.4)$ & $0(0.0)$ & $2(13.3)$ & $5(\mathrm{II} .4)$ & $7(20.6)$ & $23(12.7)$ \\
\hline $\begin{array}{l}\text { Did you miss a meeting, a work appointment } \\
\text { or did you not finish a task on time? } n(\%)\end{array}$ & I 7 (25.4) & $2(9.5)$ & $3(20.0)$ & $4(9.1)$ & $7(20.6)$ & 33 (18.2) \\
\hline $\begin{array}{l}\text { Average numbers of times going late to } \\
\text { work per patient per year }{ }^{\mathrm{a}}\end{array}$ & 2.3 & 0.6 & 0.7 & 0.7 & 0.9 & 1.2 \\
\hline $\begin{array}{l}\text { Average numbers of times leaving work } \\
\text { early per patient per year }{ }^{\mathrm{a}}\end{array}$ & 1.9 & 1.3 & 0.7 & 0.8 & 1.3 & 1.3 \\
\hline $\begin{array}{l}\text { Average numbers of times missing a full day } \\
\text { per patient per year }{ }^{a}\end{array}$ & 1.0 & 0.0 & 0.7 & 0.7 & 1.0 & 0.8 \\
\hline $\begin{array}{l}\text { Average numbers of times miss a meeting, a } \\
\text { work appointment or did not finish a task on } \\
\text { time per patient per year }{ }^{\mathrm{a}}\end{array}$ & 1.8 & 0.6 & I.I & 0.5 & 1.0 & I.I \\
\hline
\end{tabular}

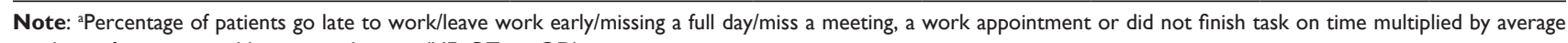
numbers of memory problem events by type (UF, QT, or QD) per patient per year.

Abbreviations: SD, standard deviation; UF, unintentionally forgetting; QT, questioning whether or not one took dose; QD, questioning how much was taken. 
Table I I Impact of memory problems on contact with health care professionals (HCPs)

\begin{tabular}{|c|c|c|c|c|c|c|}
\hline Unintentionally forgetting & $\begin{array}{l}\text { People's } \\
\text { Republic of China } \\
\mathbf{n}=\mathbf{2 3 0}\end{array}$ & $\begin{array}{l}\text { Germany } \\
n=104\end{array}$ & $\begin{array}{l}\text { USA } \\
n=|8|\end{array}$ & $\begin{array}{l}\text { UK } \\
n=190\end{array}$ & $\begin{array}{l}\text { Canada } \\
n=155\end{array}$ & $\begin{array}{l}\text { Total } \\
\mathrm{n}=860\end{array}$ \\
\hline $\begin{array}{l}\text { This last time, did you go to see your physician, or other } \\
\text { health care professional, because of missing, or because of } \\
\text { how you felt due to missing, this dose? Yes, } n(\%)\end{array}$ & $85(37.0)$ & $10(9.6)$ & $20(11.0)$ & $38(20.0)$ & $26(16.8)$ & $179(20.8)$ \\
\hline $\begin{array}{l}\text { Did you call or email your physician, or other health care } \\
\text { professional, because of missing, or because of how you } \\
\text { felt due to missing, this dose? Yes, } n(\%)\end{array}$ & $86(37.4)$ & $10(9.6)$ & $23(12.7)$ & $45(23.7)$ & $37(23.9)$ & $201(23.4)$ \\
\hline Number of visits to HCPs per patient per year ${ }^{a}$ & 3.0 & 0.7 & 1.1 & 1.5 & 1.4 & 1.7 \\
\hline $\begin{array}{l}\text { Number of contacts (calls or e-mails) to HCPs per } \\
\text { patient per year }{ }^{\mathrm{a}}\end{array}$ & 3.1 & 0.7 & 1.3 & 1.8 & 2.0 & 1.9 \\
\hline $\begin{array}{l}\text { Number of HCP minutes spent on visits per patient } \\
\text { per year }\end{array}$ & 30.0 & 7.0 & II.I & 15.0 & 14.0 & 17.1 \\
\hline $\begin{array}{l}\text { Number of HCP minutes spent on contacts (calls or } \\
\text { e-mails) per patient per year }{ }^{b}\end{array}$ & 15.5 & 3.5 & 6.4 & 9.0 & 10.0 & 9.6 \\
\hline Questioning whether or not took dose & $\begin{array}{l}\text { People's } \\
\text { Republic of China } \\
\mathrm{n}=96\end{array}$ & $\begin{array}{l}\text { Germany } \\
\mathrm{n}=56\end{array}$ & $\begin{array}{l}\text { USA } \\
n=86\end{array}$ & $\begin{array}{l}\text { UK } \\
n=86\end{array}$ & $\begin{array}{l}\text { Canada } \\
n=93\end{array}$ & $\begin{array}{l}\text { Total } \\
\mathrm{n}=417\end{array}$ \\
\hline $\begin{array}{l}\text { This last time, did you go to see your physician, or other } \\
\text { health care professional, because of missing, or because of } \\
\text { how you felt due to missing, this dose? Yes, } n(\%)\end{array}$ & $33(34.4)$ & $2(3.6)$ & $9(10.5)$ & $7(8.1)$ & $10(10.8)$ & $61(14.6)$ \\
\hline $\begin{array}{l}\text { Did you call or email your physician, or other health care } \\
\text { professional, because of missing, or because of how you } \\
\text { felt due to missing, this dose? Yes, } n(\%)\end{array}$ & $33(34.4)$ & $3(5.4)$ & $12(14.0)$ & $8(9.3)$ & II (II.8) & $67(16.1)$ \\
\hline Number of visits to HCPs per patient per year & 2.4 & 0.3 & 0.8 & 0.6 & 0.9 & 1.1 \\
\hline $\begin{array}{l}\text { Number of contacts (calls or e-mails) to HCPs per } \\
\text { patient per year }\end{array}$ & 2.4 & 0.4 & 1.1 & 0.6 & 1.0 & 1.2 \\
\hline $\begin{array}{l}\text { Number of HCP minutes spent on visits per patient } \\
\text { per year }{ }^{b}\end{array}$ & 24.0 & 3.0 & 8.0 & 6.0 & 9.0 & 11.0 \\
\hline $\begin{array}{l}\text { Number of HCP minutes spent on contacts (calls or } \\
\text { e-mails) per patient per year }\end{array}$ & 12.0 & 2.0 & 5.5 & 3.0 & 5.0 & 6.0 \\
\hline Questioning how much was taken & $\begin{array}{l}\text { People's } \\
\text { Republic of China } \\
\mathbf{n}=74\end{array}$ & $\begin{array}{l}\text { Germany } \\
\mathrm{n}=29\end{array}$ & $\begin{array}{l}\text { USA } \\
n=33\end{array}$ & $\begin{array}{l}\text { UK } \\
n=50\end{array}$ & $\begin{array}{l}\text { Canada } \\
\mathrm{n}=49\end{array}$ & $\begin{array}{l}\text { Total } \\
\mathrm{n}=235\end{array}$ \\
\hline $\begin{array}{l}\text { This last time, did you go to see your physician, or other } \\
\text { health care professional, because of missing, or because of } \\
\text { how you felt due to missing, this dose? Yes, } n(\%)\end{array}$ & $28(37.8)$ & $4(13.8)$ & $3(9.1)$ & $8(16.0)$ & $7(14.3)$ & $50(21.3)$ \\
\hline $\begin{array}{l}\text { Did you call or email your physician, or other health care } \\
\text { professional, because of missing, or because of how you } \\
\text { felt due to missing, this dose? Yes, } n(\%)\end{array}$ & $32(43.2)$ & $2(6.9)$ & $5(\mid 5.2)$ & $9(18.0)$ & $10(20.4)$ & $58(24.7)$ \\
\hline Number of visits to HCPs per patient per year ${ }^{a}$ & 2.7 & 0.9 & 0.5 & 1.0 & 0.7 & 1.3 \\
\hline $\begin{array}{l}\text { Number of contacts (calls or e-mails) to HCPs per } \\
\text { patient per year }\end{array}$ & 3.1 & 0.5 & 0.9 & I.I & 1.0 & 1.5 \\
\hline $\begin{array}{l}\text { Number of HCP minutes spent on visits per patient } \\
\text { per yearb }\end{array}$ & 27.0 & 9.0 & 5.1 & 10.0 & 7.0 & 13.2 \\
\hline $\begin{array}{l}\text { Number of HCP minutes spent on contacts (calls or } \\
\text { e-mails) per patient per year }{ }^{\mathrm{b}}\end{array}$ & 15.5 & 2.5 & 4.3 & 5.5 & 5.0 & 7.7 \\
\hline
\end{tabular}

Notes: aPercentage of patients go to see/call or email physician or other health care professional multiplied by average numbers of memory problem events by type (UF, QT, or QD) per patient per year; ' l't is assumed that 10 minutes is spent per HCP visit, 5 minutes is spent per HCP contact (call or e-mail).

Abbreviations: UF, unintentional forgetting; QT, questioning whether or not one took dose; QD, questioning how much was taken.

more likely to skip a dose following UF (34.4\%) compared with subjects with T1D $(23.8 \%)$ and were less likely to measure blood glucose (T2D, 44.1\%; T1D, 48.4\%) or take a dose without measuring blood glucose (T2D, 21.5\%; T1D, 27.8\%) than subjects with T1D $(P<0.001)$. Further, subjects with T2D were significantly less likely to contact/visit a health care professional $(26.2 \%)$ than subjects with T1D $(35.3 \%$, $P<0.01)$.

Further, duration of disease also had a significant association with the results for UF, including frequency of UF, corrective action taken following UF, missed work time, and health care contact. Those who "never" experienced UF had 
a significantly higher mean duration of diabetes (15.1 years) compared with those who experienced UF more frequently ("very rarely/rarely", 11.4 years; "sometimes", 9.6 years; "often/very often", 11.7 years; $P<0.001)$. Those who took a dose of insulin without measuring blood glucose following UF also had a higher average duration of diabetes (13.7 years) compared with those who measured blood glucose (9.5 years) or skipped a dose (11.9 years, $P<0.001)$. Those who did not miss work time had a greater average duration of diabetes (11.2 years) than those who did miss work time (9.3 years, $P<0.05)$. Further, those who did not contact health care professionals had a greater average duration of diabetes (11.9 years) compared with those who did $(10.0$ years, $P<0.01)$.

\section{Questioning whether or not a dose was taken}

Some results for QT also varied significantly by age, type of diabetes, and duration of diabetes. Respondents who reported that they "never" experienced QT had a significantly higher mean age (45.1 years) than those who reported experiencing QT more frequently ("very rarely, rarely", 40.3 years; "sometimes", 40.2 years; "often/very often", 41.8 years, $P<0.001)$. For corrective action, those who skipped a dose following QT had a significantly higher mean age (46.1 years) than those who measured blood glucose (39.8 years) and those who took a dose without measuring blood glucose (38.9 years, $P<0.001$ ). Also, respondents who missed work time were significantly younger (35.4 years) than those who did not miss work (38.6 years, $P<0.05$ ). Further, those who contacted health care professionals were significantly younger (37.0 years) than those who did not (43.5 years, $P<0.001$ ).

Type of diabetes was associated with both frequency of QT and corrective action following QT. Patients with T2D were significantly more likely to report that they "never" experienced QT (14.4\%) than those with T1D (8.2\%). On the other hand, subjects with T1D were significantly more likely to indicate that they "sometimes" experienced QT $(23.0 \%)$ compared with those with T2D $(17.0 \%, P<0.001)$. In terms of corrective action, subjects with T2D were significantly more likely to skip a dose $(45.0 \%)$ than subjects with T1D (34.3\%). Patients with T1D, on the other hand, were more likely to take a dose without measuring blood glucose $(27.4 \%)$ than those with T2D $(17.1 \%, P<0.05)$. Both types were about equally likely to measure blood glucose and take insulin based on a reading (T1D, 38.3\%; T2D, 37.8\%).

Duration of diabetes was significantly associated with corrective action and health care contact following QT. Those who measured blood glucose following QT had a significantly shorter duration of diabetes ( 8.7 years) than those who skipped a dose following QT (12.2 years) or took insulin without measuring blood glucose $(11.9$ years, $P<0.001)$. Moreover, those who contacted health care professionals as a result of QT had a significantly shorter duration of diabetes (8.2 years) than those who did not (11.5 years, $P<0.01)$.

\section{Questioning amount of dose taken}

Some of the findings for QD also varied significantly by age, diabetes type, and duration of diabetes. Age was associated with both frequency of QD and health care contact. Respondents who reported that they "never" experienced QD were older on average ( 44.7 years) than those who experienced QD more frequently ("very rarely/rarely", 39.6 years; "sometimes", 38.5 years; "'often/very often", 38.5 years, $P<0.001$ ). Also, respondents who contacted health care professionals following QD were younger on average (37.6 years) compared with those who did not ( 41.8 years, $P<0.05$ ).

Diabetes type was also related to the frequency of experiencing QD. Specifically, subjects with T1D reported experiencing QD more frequently than those with T2D, while subjects with T2D were significantly more likely to report that they "never" experienced QD (38.1\%) than those with T1D $(23.8 \%, P<0.001)$.

Further, duration of diabetes was associated with both frequency of QD and corrective actions following QD. Respondents who reported experiencing QD “never" or "very rarely/rarely" had a significantly longer duration of diabetes ("never", 12.4 years; "very rarely/rarely", 11.5 years) compared with those who reported experiencing QD more frequently ("sometimes", 9.9 years; "often/very often", 9.8 years, $P<0.01)$. Additionally, those who measured blood glucose and took insulin based on a reading following QD had a significantly shorter average duration of diabetes ( 9.6 years) than those who took insulin without measuring blood glucose (13.1 years) or skipped a dose $(12.7$ years, $P<0.05)$.

\section{Insulin regime}

Analyses revealed few significant differences in results by type of insulin regime, the most important of which are described below. Respondents using the basal bolus regime were more likely to report that they experienced UF $(72.9 \%)$ than those using basal only $(60.0 \%)$ or premix $(64.4 \%$, $P<0.001)$. Respondents using a basal bolus or premix regime were more likely to experience QD than those using a basal only regime (basal bolus, $57.4 \%$; basal only, $46.9 \%$; premix, $56.7 \%, P<0.05)$. In terms of confidence in knowing what to do following QT and QD, the basal only group was significantly less likely (QT, $P<0.001$; QD, $P<0.01$ ) to report 
being "very/extremely" confident (QT, 25.6\%; QD, 22.2\%) compared with the basal bolus (QT, 40.7\%; QD, 35.4\%) and premixed (QT, 35.1\%; QD, 37.8\%) groups. This may be due to respondents on basal bolus or premixed regimes having had diabetes for a longer period of time. For all three MPs, respondents on a basal bolus regime were more likely to report that a routine blood glucose measurement led to their realization of the MP compared with those taking a basal only or premix regime. This may be due to those with the basal bolus regime having more opportunities to measure blood glucose.

In terms of corrective action taken following QT, those on a basal bolus regime were more likely to skip an insulin dose and be less likely to take an insulin dose without measuring blood glucose compared with the basal only and premixed groups. Additionally, those on the premix regime reported a significantly shorter average recovery time for returning to normal blood glucose levels following QD than the basal only and basal bolus groups. Respondents taking the premix regime were also more likely than those on the other regimes to report being late for work following QD. Those on the basal bolus regime were more likely to experience hyperglycemia following QT compared with those on the other insulin regimes. Finally, respondents on the basal only regime were more likely to call/email a physician or health care professional following QD than respondents on other regimes.

\section{Discussion}

These results demonstrate that MPs related to insulin-taking are prevalent among diabetes patients across the globe and that MPs impact more than simply missing a dose. This high incidence of MPs among people with diabetes is an important new finding that adds to our knowledge about insulin-taking behavior. While $68 \%$ of patients reported a UF in the past month, even more patients (79.6\%) reported a QT in the past month and 54.7\% reported a QD in the past month. Most patients (66.5\%) reported having more than one MP, and of the three MPs, QT was the most prevalent. It is also clear from these findings that the impact of MPs is not inconsequential, and that these impacts are not, for the most part, influenced by type of regime, although there is some variation in results by age, type of diabetes, and duration of disease. Of further note is that $15 \%$ of the sample reported having MPs that were not medically diagnosed and may have affected their frequency of MPs. However, given that the sample was intended to represent the general population of people with diabetes and without known memory issues due to another medical condition such as dementia, we believe that inclusion of these respondents in our sample allows for the findings to be more representative of the real world of diabetes care.

Overall, the findings lead to a better understanding of the experience of diabetes patients concerning MPs related to the taking of insulin. We found that most patients become aware of MPs because they just did not "feel right". Moreover, patients were most likely to be relaxing or waking up in the morning when they experienced an MP. Further, being busy and being tired, followed by major disruptions, were the most common reasons given for occurrence of MPs. Such information might be used by clinicians to help patients better understand when and where MPs are likely to occur in order to prevent them or address them in a timely manner.

The study also demonstrates that MPs related to insulintaking have serious impacts on diabetes management. The majority of respondents expressed concern about experiencing hyperglycemia following an MP, and many reported being concerned about diabetes complications in the future. Indeed, about half of respondents for each MP indicated that they actually experienced hyperglycemia as a consequence of their MP (UF, 58.4\%; QT, 48.7\%; QD, 46.4\%). This has important implications because research shows that hyperglycemia is associated with decreased immune function, cardiovascular symptoms including increased blood pressure and heart rate, thrombosis, vascular problems related to cell inflammation, central nervous system injury, increased hospital admission and length of stay, and increased mortality. ${ }^{12}$

Respondents also expressed moderate degrees of worry when considering which corrective action to take following an MP. While between 38\% (QT) and 46\% (UF) of respondents measured blood glucose and took action based on the reading, many respondents took insulin without measuring blood glucose or skipped a dose, essentially doing nothing. Additionally, about a quarter of patients indicated that they were not at all or only a little confident in knowing what to do when they experienced an MP (UF, 29\%; QT, 25\%; QD, 26\%). This finding strongly suggests that patients would benefit from increased patient education on how to handle MPs associated with insulin-taking.

The results also show that average recovery times following MPs were not brief or fleeting, particularly for UF. Patients who experienced a UF indicated longer recovery times compared with those who experienced a QT or QD. This is not surprising given that those who simply question a dose may have actually taken the dose or the correct amount. 
Nevertheless, QT and QD still have important implications for patients' well-being. As the study showed, respondents reported that all three MPs had moderate negative impacts on respondents' physical and emotional functioning, on average.

Beyond impacts on the individual patient, the results showed that MPs related to insulin-taking have implications for patients' family members. Indeed, between 32\% (QD) and $39 \%$ (UF) of patients indicated that causing family members to worry was a concern they had related to their MP. Thus, the impact of patient MPs clearly extends beyond the individual, even for simply questioning a dose. Future research should explore further the impact of treatment of diabetes on patients' family members.

In addition to the impact on individual patients and their families, the results also suggest that MPs among patients have economic impacts globally. The study demonstrates that MPs have an adverse effect on time at work, including being late to work, leaving early, missing meetings, or missing a full day. Indeed, between 15\% (QT) and 20\% (UF) of respondents reported being at work when their MPs occurred, and a substantial number reported missing work time as a result of MPs. Additionally, respondents reported that MPs led to additional blood glucose measurements, leading to greater costs for blood glucose testing strips. Further, MPs are associated with increased health care costs, as many patients reported visiting or contacting physicians or other health care professionals due to MPs.

Beyond direct economic impacts, the results suggest that MPs related to insulin-taking have additional intangible economic impacts. Respondents reported that MPs had moderate negative impacts on patient-reported physical and emotional functioning. Patients also reported moderate negative impacts of MPs on physical and emotional functioning at work, which has implications for worker productivity, because workers may not be functioning at their optimal levels following an MP. Further, the impact on family members may be another intangible cost of MPs, and is not well understood.

As expected, the analyses revealed significant differences in the patient experience of MPs and their impact by country. In particular, the experience and impact of MPs among diabetic patients in the People's Republic of China were in many cases different from those of diabetic patients from other countries in the sample. Some of these differences may be explained by differences in cultural and health care beliefs and practices in the People's Republic of China compared with other countries in the survey. For example, more MPs occurred at work in the Chinese subjects. This difference may be explained by the fact that the Chinese spend a greater proportion of their time at work. Additional analyses suggest that type of insulin regime is not the source of these differences between the People's Republic of China and other countries. Further research is needed to better understand this cross-country variation.

The results show some significant differences across demographic and disease-specific factors, including age, type of diabetes, and duration of diabetes. While age was associated with a lower frequency of QT and QD, it was associated with a greater frequency of UF. Age was also positively associated with skipping a dose following both UF and QT. Further, respondents who missed work time due to UF and QT were significantly younger on average than those who did not miss work time. For all three MPs, respondents who contacted a health care professional following the MP were significantly younger than those who did not. There were also some significant differences in results by type of diabetes. T2D subjects reported experiencing QT and QD less frequently compared with T1Ds. Additionally, T2D subjects were more likely to skip a dose following UF and QT compared with T1D subjects. T2D subjects were also less likely to contact a physician/health care professional following UF. The results also revealed some significant differences by duration of disease. Duration of disease was negatively associated with frequency of UF and QD. Also, respondents who missed work time due to UF had significantly lower duration of disease compared with those who did not miss work time. Respondents who contacted a health care professional following UF and QT also had a lower duration of disease than those who did not contact a health care professional. These findings suggest that some targeted educational interventions for certain subgroups of patients may be warranted, specifically subjects with T2D at the time of diagnosis.

The web survey found that MPs were generally not related to type of regimen. Although there were some significant differences across type of regime, there was no consistent pattern of difference. This is expected because MPs are more likely to be inherent in individuals rather than the insulin regime they are taking. In the limited instances in which the basal bolus regimen was associated with a greater incidence of MPs, this may be explained by the basal bolus regime allowing more opportunities to forget, given the greater complexity of the regimen compared with the basal only and premix regimes. Indeed, research suggests that multiple medications, regimen complexity, and frequent dosing are associated with medication nonadherence in diabetes patients. ${ }^{4,7,13}$ 
Finally, additional analyses showed that blood glucose measurement was associated with a greater likelihood of both experiencing hyperglycemia and missing time at work. These results may be due to the fact that those who are experiencing more severe impacts are in reality the ones who did not take their dose when having a QT or took the incorrect dose when having a QD. Thus, the MP-related nonadherence resulted in feeling the ill effects of poor glucose control and led these respondents to assess their blood glucose in response to their symptoms. Given the high prevalence and incidence of MPs, and that for QT $50 \%$ of patients did not use a memory aid or used one and still were not sure if they had taken their dose, all patients would benefit from a "memory aid", which would give them accurate and reliable information as to whether or not the MP resulted in nonadherence. However, these truly nonadherent patients are the group who would likely benefit most from having a "memory aid" that would give them accurate information regarding their insulin adherence in a more timely manner. With this information, patients may then be more able to reduce the number and/or frequency of additional blood glucose monitoring and take corrective action sooner before negative consequences occur.

While the study makes important contributions to the understanding of MPs and insulin-taking behavior among diabetes patients, some limitations should be recognized. There is some potential for selection bias, as the survey was conducted online. In the People's Republic of China, for example, it is estimated that there were 389 million Internet users in $2009^{14}$ in a population of about 1.3 billion at that time, ${ }^{15}$ so a proportion of the population was not able to complete the online survey, and it is possible that MPs and insulintaking behaviors are different in this population. Additionally, the study was based on patient self-reports, so potential recall bias may have been an issue affecting our results, given that patients were asked to report on past incidents. However, patients were only asked to report on incidents in the past month, so it is likely that recall bias was somewhat limited. Social desirability bias may have influenced responses, as patients are more likely to give more positive assessments of their insulin-taking behavior. The web survey format, which allows for complete anonymity of the respondent, may have limited this potential source of bias.

\section{Conclusion}

This study contributes to our knowledge about insulin-taking behavior and MPs among people with diabetes globally. The results demonstrate that MPs associated with insulintaking are quite common, and this is the first study to explore such MPs in depth. The findings shed light on the experiences of MPs in diabetes patients from five countries, as well as the consequences of MPs, which are not trivial. Overall, there is some variation in the experiences and impacts of MPs by age, type of diabetes, and duration of disease, but few differences across types of insulin regime. Some cross-country differences in results were evident, with results from the People's Republic of China frequently differing significantly from the results of other countries in the study. Further research is needed to understand these differences. The findings suggest that MPs should be considered in the treatment of diabetes in order to improve insulin adherence, diabetes management, and patient outcomes.

\section{Disclosure}

$\mathrm{MB}$ and KMP are paid consultants to Novo Nordisk A/S. RS, $\mathrm{KMC}$, and JHK are paid employees of Novo Nordisk A/S. The authors report no other conflicts of interest in this work.

\section{References}

1. Cramer JA. A systematic review of adherence with medications for diabetes. Diabetes Care. 2004;27(5):1218-1224.

2. Cramer JA, Benedict A, Muszbek N, Keskinaslan A, Khan ZM. The significance of compliance and persistence in the treatment of diabetes, hypertension and dyslipidaemia: a review. Int J Clin Pract. 2008;62(1): 76-87.

3. Brod M, Rana A, Barnett AH. Adherence patterns in patients with type 2 diabetes on basal insulin analogues: missed, mistimed and reduced doses. Curr Med Res Opin. 2012;28(12):1933-1946.

4. Donnelly LA, Morris AD, Evans JM. Adherence to insulin and its association with glycaemic control in patients with type 2 diabetes. QJM. 2007;100(6):345-350.

5. Peyrot M, Barnett AH, Meneghini LF, Schumm-Draeger PM. Insulin adherence behaviours and barriers in the multinational Global Attitudes of Patients and Physicians in Insulin Therapy study. Diabet Med. 2012;29(5):682-689.

6. Peyrot M, Barnett AH, Meneghini LF, Schumm-Draeger PM. Factors associated with injection omission/non-adherence in the Global Attitudes of Patients and Physicians in Insulin Therapy study. Diabetes Obes Metab. 2012;14(12):1081-1087.

7. Peyrot M, Rubin RR, Kruger DF, Travis LB. Correlates of insulin injection omission. Diabetes Care. 2010;33(2):240-245.

8. Cramer JA, Pugh MJ. The influence of insulin use on glycemic control: how well do adults follow prescriptions for insulin? Diabetes Care. 2005;28(1):78-83.

9. Randlov J, Poulsen JU. How much do forgotten insulin injections matter to hemoglobin A1c in people with diabetes? A simulation study. J Diabetes Sci Technol. 2008;2(2):229-235.

10. Currie CJ, Peyrot M, Morgan CL, et al. The impact of treatment noncompliance on mortality in people with type 2 diabetes. Diabetes Care. 2012;35(6):1279-1284.

11. Morris AD, Boyle DI, McMahon AD, Greene SA, MacDonald TM, Newton RW. Adherence to insulin treatment, glycaemic control, and ketoacidosis in insulin-dependent diabetes mellitus. The DARTS/MEMO Collaboration. Diabetes Audit and Research in Tayside Scotland. Medicines Monitoring Unit. Lancet. 1997;350(9090):1505-1510.

12. Clement S, Braithwaite SS, Magee MF, et al. Management of diabetes and hyperglycemia in hospitals. Diabetes Care. 2004;27(2): 553-591. 
13. Bailey CJ, Kodack M. Patient adherence to medication requirements for therapy of type 2 diabetes. Int J Clin Pract. 2011;65(3):314-322.

14. World Factbook. People's Republic of China [annual on the Internet]. Washington, DC, USA: US Central Intelligence Agency; 2013. Available from: https://www.cia.gov/library/publications/the-world-factbook/ geos/ch.html. Accessed June 25, 2013.
15. World Population Data Sheet [report on the Internet]. Washington, DC, USA: Population Reference Bureau; 2009. Available from: http://www. prb.org/pdf09/09wpds_eng.pdf. Accessed June 25, 2013.

\section{Publish your work in this journal}

Patient Intelligence is an international, peer-reviewed, open access journal that characterizes and measures the central role of patient behavior and intention in optimizing healthcare management in all areas of disease and complaint types. An improved understanding of patient intelligence coupled with predictive analysis helps an organization contribute more effectively to achieving better outcomes.

Submit your manuscript here: http://www.dovepress.com/patient-intelligence-journal

\section{Dovepress}

The journal is characterized by the rapid reporting of reviews, original research, methodologies, analytics, modeling, clinical studies and patient surveys across all disease areas. The manuscript management system is completely online and includes a very quick and fair peer-review system. Visit http://www.dovepress.com/ testimonials.php to read real quotes from published authors. 